\title{
Shearer's point process, the hard-sphere model and a continuum Lovász Local Lemma
}

\author{
Hofer-Temmel Christoph (math@temmel.me)
}

\begin{abstract}
A point process is R-dependent, if it behaves independently beyond the minimum distance $\mathrm{R}$. This work investigates uniform positive lower bounds on the avoidance functions of R-dependent simple point processes with a common intensity. Intensities with such bounds are described by the existence of Shearer's point process, the unique R-dependent and Rhard-core point process with a given intensity. This work also presents several extensions of the Lovász Local Lemma, a sufficient condition on the intensity and $\mathrm{R}$ to guarantee the existence of Shearer's point process and exponential lower bounds. Shearer's point process shares combinatorial structure with the hard-sphere model with radius $\mathrm{R}$, the unique R-hardcore Markov point process. Bounds from the Lovász Local Lemma convert into lower bounds on the radius of convergence of a high-temperature cluster expansion of the hard-sphere model. This recovers a classic result of Ruelle on the uniqueness of the Gibbs measure of the hard-sphere model via an inductive approach à la Dobrushin.
\end{abstract}

Keywords: avoidance function ; Lovász Local Lemma ; hard-sphere model ; partition function ; R-dependent point process ; R-hard-core MSC(2010): 60G55 (60G60; 82B05; 05C69)

\section{Contents}

1 Introduction

2 Results 3

2.1 Uniform bounds on the avoidance functions . . . . . . . . . B

2.2 The generating function $\ldots \ldots \ldots \ldots \ldots \ldots$

2.3 Shearer's point process . . . . . . . . . . . . . . . . . . 4

2.4 The hard-sphere model . . . . . . . . . . . . . . . . 5

2.5 Sufficient conditions for exponential bounds . . . . . . . . 5

2.6 Stochastic domination and order . . . . . . . . . . 7

3 About one-dependent PPs 8

4 Properties of the generating function 10

4.1 Basic properties . . . . . . . . . . . . . . . . 10

4.2 Cluster expansion and monotonicity . . . . . . . . . 12

4.3 Continuity properties . . . . . . . . . . . . . . 12

4.4 Behaviour under the set difference operator . . . . . . . . . . 14 
5 Proofs around Shearer's point process

5.1 Characterisation, uniqueness and existence. . . . . . . . . 16

5.2 Proof of Theorem 4. . . . . . . . . . . . . . . . 19

5.3 Proof of Theorem 6. . . . . . . . . . . . . . . . 19

5.4 Intrinsic coupling and monotonicity . . . . . . . . . . . . 20

5.5 Shearer's PP is different . . . . . . . . . . . . . . . . . 21

6 Proofs of the LLLs 22

7 Additional material 24

7.1 Association and ordering properties ............ 24

7.2 Miscellaneous proofs . . . . . . . . . . . . . 25

7.3 Direct proof of homogeneous LLL in Euclidean space . . . . . . . 26

7.4 Inductive proofs . . . . . . . . . . . . . . 27 27

\section{Introduction}

A point process $(\mathrm{PP}) \xi$ on a complete separable metric space is $R$-dependent, if events of $\xi$ based on Borel sets having mutual distance greater than or equal to $R$ are independent. This work only deals with simple PPs. Natural examples of $R$ dependent PPs are as follows. A Poisson PP, which is even 0-dependent. Range $R / 2$ dependent thinnings of Poisson PPs à la Matérn [26, 35]. Poisson cluster PPs [4, 10] with an offspring distribution supported on a sphere of radius $R / 2$ around a cluster centre point. Local constructions based on a Poisson PP, such as taking the centres of circumscribed circles of radius less than $R / 2$ of triangles formed by triples of points from the Poisson PP. Determinantal and permanental PPs 6, 15, 34, with a kernel of bounded range $R$ are also $R$-dependent.

If the space is discrete, then a simple $\mathrm{PP}$ is a Bernoulli random field (short $\mathrm{BRF})$, an at most countable collection of $\{0,1\}$-valued random variables indexed by the space. The study of $R$-dependent BRFs has a long history in the theory of discrete stochastic processes [1, 2, 17, 8, 12, 13, 22, 25]. Further uses of $R$-dependent BRFs are within the part of the probabilistic method in combinatorics building on the Lovász Local Lemma (LLL) [3, 16] and in graph colouring [21, 23].

Let $(\mathcal{X}, \delta)$ be the complete separable metric space. Without loss of generality, rescaling the metric reduces the discussion to 1 -dependence. Let $\mathcal{B}^{b}$ and $\mathcal{B}$ be the set of bounded and all Borel sets on $\mathcal{X}$ respectively. Let $\mathcal{M}^{b}$ be the space of boundedly finite Borel measures. The intensity measure of a PP is the expected number of points in a given Borel set $B$. For $M \in \mathcal{M}^{b}$, let $\mathcal{C}(M)$ be the class of simple and 1-dependent PP laws with intensity measure $M$. Where there is no danger of confusion, identify a PP and its law. For $\xi \in \mathcal{C}(M)$ and $B \in \mathcal{B}^{b}$, the avoidance probability is the probability of $\xi$ having no points in $B$. The avoidance function of $\xi$ maps $\mathcal{B}^{b}$ to the avoidance probabilities of $\xi$.

This paper studies uniform lower bounds on the avoidance function of PPs in $\mathcal{C}(M)$. First, Section 2.1 extends a dichotomy by Shearer 33 from BRFs to PPs. For large intensity measure $M$, there exists a $\mathrm{PP}$ in $\mathcal{C}(M)$ with an avoidance function vanishing on some bounded Borel set of positive $M$-measure (zero phase). For small intensity measure $M$, there is a uniform positive lower bound on the avoidance function of PPs in $\mathcal{C}(M)$ (positive phase). 
Second, for $M$ in the positive phase, Section 2.3 generalises a construction by Shearer 33 of the unique $\mathrm{PP}$ in $\mathcal{C}(M)$ with minimal avoidance function. A set of points is $r$-hard-core, if its points have mutual distance at least $r$. A $\mathrm{PP} \xi$ is $r$-hard-core, if its realisations are almost-surely so. An $r$-hard-core and $R$-dependent $\mathrm{PP}$ must have $r \leq R$. $R$-dependence and $R$-hard-core together imply uniqueness of the PP law and existence only for small intensity measures. If a PP with these properties exists, call it Shearer's PP. Shearer's PP has the minimal avoidance function in $\mathcal{C}(M)$, because it avoids clusters of points and spreads its points all over space. The existence of Shearer's PP describes the positive phase.

Section 2.4 recalls the hard-sphere model, the unique Markov PP with range $R$ interaction and an $R$-hard-core. The partition function of the hard-sphere model and the avoidance function of Shearer's PP have the same algebraic structure. Shearer's PP exists for a given intensity measure $M$, if and only if the cluster expansion of the hard-sphere model converges uniformly and absolutely at negative fugacity $-M$, generalising the identification in the BRF case 32.

Third, Section 2.5 generalises the LLL [16] to the case of PPs. Here, a LLL denotes a sufficient condition on $\mathcal{X}$ and $M$ for a uniform exponential lower bound on the avoidance functions in $\mathcal{C}(M)$ and to be in the positive phase. The core idea is to derive global properties, i.e., being in the positive phase, from local properties. On $\mathbb{R}^{d}$, this yields an explicit and uniform upper bound on the empty space functions $F$ [11, Section 15.1] and $J$ functions [38] of isotropic and 1dependent PPs. For the hard-sphere model, a LLL becomes a sufficient condition for the convergence of the cluster expansion and yields a lower bound on the radius of convergence. This improves a classic lower bound via cluster expansion techniques by Ruelle [31] by a short inductive argument à la Dobrushin [14] of less than two pages.

Section 2.6 explains the relevance of exponential lower bounds on the avoidance function for uniform stochastic domination by a Poisson PP. Section 3 discusses variations of 1-dependence and a key inequality of the avoidance functions of 1-dependent PPs. The remaining sections contain proofs.

\section{Results}

\subsection{Uniform bounds on the avoidance functions}

The first question is the existence of 1-dependent PPs with a given intensity measure. Let $\mathcal{M}^{\emptyset}:=\left\{M \in \mathcal{M}^{b} \mid \mathcal{C}(M)=\emptyset\right\}$. Proposition 15 shows that atoms of mass greater than one in the intensity measure are the only obstacle. Thus, $\mathcal{C}(M) \neq \emptyset$, if and only if $M \in \mathcal{M}^{b} \backslash \mathcal{M}^{\emptyset}=\left\{M \in \mathcal{M}^{b} \mid \forall x \in \mathcal{X}: M(\{x\}) \leq 1\right\}$.

This work is about lower bounds on the avoidance functions of 1-dependent PPs. The first result extends a dichotomy by Shearer 33 from BRFs to PPs.

Theorem 1. If $M \in \mathcal{M}^{b} \backslash \mathcal{M}^{\emptyset}$, then it falls into one of two phases. In the zero phase, there is a $\xi \in \mathcal{C}(M)$ with zero avoidance probability on some $B \in \mathcal{B}^{b}$ of positive measure. That is, $M(B)>0$ and $\mathbb{P}(\xi(B)=0)=0$. In the positive phase, there is a unique $\mu \in \mathcal{C}(M)$ minimizing the (conditional) avoidance probabilities uniformly in space and the class. That is, for every $\xi \in \mathcal{C}(M)$ and all $A, B \in \mathcal{B}^{b}$, one has

$$
\mathbb{P}(\xi(B)=0 \mid \xi(A)=0) \geq \mathbb{P}(\mu(B)=0 \mid \mu(A)=0)>0 .
$$


Theorem 1 follows from Corollary 5 and Theorem 6. Section 2.3 describes the distinguished PP $\mu$ in the positive phase. Let $\mathcal{M}^{0}$ and $\mathcal{M}^{+}$be the subsets of $\mathcal{M}^{b} \backslash \mathcal{M}^{\emptyset}$ being in the zero phase and positive phase respectively. See also Figure 1 .

\subsection{The generating function}

A configuration is a countable collection of points in $\mathcal{X}$. A configuration $C$ is 1-hard-core, if its points have mutual distance at least one, i.e., for all $\{x, y\} \subseteq$ $C: \delta(x, y) \geq 1$. In the classic case of the metric space $(V, 2 d)$ derived from a graph $G:=(V, E)$ with geodesic metric $d$, the 1-hard-core configurations are the graph-theoretic independent sets of $G$. For $n \in \mathbb{N}_{0}$, let $h_{n}$ be the indicator function of 1-hard-core tuples in $\mathcal{X}^{n}$.

Definition 2. The generating function $Z$ of weighted 1-hard-core configurations is

$\mathcal{B}^{b} \times \mathcal{M}^{b} \rightarrow \mathbb{R}$ and

$$
(B, M) \mapsto \sum_{n=0}^{\infty} \frac{(-1)^{n}}{n !} \int_{B^{n}} h_{n}\left(x_{1}, \ldots, x_{n}\right) \prod_{i=1}^{n} M\left(\mathrm{~d} x_{i}\right)
$$

Proposition 16 shows that $Z$ is well-defined. If $M$ is diffuse, then $Z(B, M)$ is the expectation of a functional of a Poisson PP of intensity $M$. Such a representation fails for intensity measures containing atoms. The alternating sign in $Z(B, M)$ is a convenience to avoid using a negative measure as argument to $Z$ throughout most of this work. For unambiguous choices of $M, Z(B)$ abbreviates $Z(B, M)$. While $Z(\emptyset)=1$ always holds, a central topic is which $M$ admit $Z(B, M) \geq 0$ uniformly in $B$. For all $A, B \in \mathcal{B}^{b}$ with $Z(B)>0$, a key quantity is $z(A, B):=Z(A \cup B) / Z(B)$. Section 4 discusses the properties of $Z$ and $z$ in detail.

\subsection{Shearer's point process}

This section is about 1-dependent PPs with an 1-hard-core. For a given intensity measure, there is at most one such PP. If it exists, call it Shearer's PP, as it generalises the BRF construction of Shearer [33.

Theorem 3. If an 1-hard-core $\eta_{M} \in \mathcal{C}(M)$ exists, then its law is unique. Its avoidance function is $Z$, i.e., for each $B \in \mathcal{B}^{b}, \mathbb{P}\left(\eta_{M}(B)=0\right)=Z(B)$. Such a $P P$ exists for all intensity measures in $\mathcal{M}^{\text {sh }}:=\left\{M \in \mathcal{M}^{b} \mid \forall B \in \mathcal{B}^{b}: Z(B, M) \geq 0\right\}$.

The proofs of this section's statements are in Section 5. If Shearer's PP exists, then it minimizes the (conditional) avoidance probabilities within the class of 1-dependent PPs with the same intensity measure.

Theorem 4. If $M \in \mathcal{M}^{\text {sh }}$, then, for all $\xi \in \mathcal{C}(M)$ and $A, B \in \mathcal{B}^{b}$ with $Z(B)>0$,

$$
\mathbb{P}(\xi(A)=0 \mid \xi(B)=0) \geq z(A, B) \geq 0 .
$$

If Shearer's PP has a positive avoidance function, then it is the unique PP $\mu$ from the positive phase in Theorem 1 . Let $\mathcal{M}^{>}:=\left\{M \in \mathcal{M}^{b} \mid \forall B \in \mathcal{B}^{b}: Z(B, M)>0\right\}$. 
Corollary 5. If $M \in \mathcal{M}^{>}$, then $M \in \mathcal{M}^{+}$and Shearer's PP $\eta_{M}$ has the minimal (conditional) avoidance function in $\mathcal{C}(M)$. In short, $\mathcal{M}^{>} \subseteq \mathcal{M}^{+}$.

Corollary 5 is a direct consequence of Theorem 44. On the other hand, if Shearer's PP does not have a positive avoidance function on $\mathcal{B}^{b}$ or does not exist, then there is a PP with zero avoidance probability on some bounded Borel set. This puts the intensity measure $M$ in the zero phase.

Theorem 6. If $M \notin \mathcal{M}^{>}$and $M \notin \mathcal{M}^{\emptyset}$, then $M \in \mathcal{M}^{0}$.

Theorem 6 and Corollary 5 together imply that $\mathcal{M}^{>}=\mathcal{M}^{+}$.

Independent thinning of Shearer's PP decreases the intensity measure and preserves the two characterising properties of 1-dependence and 1-hard-core. A bigger intensity measure decreases the avoidance function or inhibits the existence of 1-dependent PPs.

Theorem 7. The sets $\mathcal{M}^{\text {sh }}$ and $\mathcal{M}^{>}=\mathcal{M}^{+}$are down-sets, i.e., closed under decreasing the measure. The set $\mathcal{M}^{\emptyset}$ is an up-set, i.e., closed under increasing the measure. The set $\mathcal{M}^{0}$ is an up-set, as long as atoms do not increase beyond mass one.

In general, Shearer's PP differs from all other $R$-independent models in the introduction. Probabilistic constructions of Shearer's PP are known only in special cases. Details are in Section 5.5.

\subsection{The hard-sphere model}

Another simple 1-hard-core PP related to the function $Z$ is the hard-sphere model. It is a Markov PP with the most repulsive range 1 interaction [31, Section 1.2.2]. A common visualisation of the hard-sphere model is as a collection of nonoverlapping open spheres with radius $1 / 2$ representing the hard cores of atoms.

The hard-sphere model $h_{B, M}$ in a finite volume $B \in \mathcal{B}^{b}$ with fugacity $M \in$ $\mathcal{M}^{b}$ and empty boundary conditions has Janossy intensity

$$
\mathbb{P}\left(h_{B, M}=\mathrm{d}\left(x_{1}, \ldots, x_{n}\right)\right)=\frac{h_{n}\left(x_{1}, \ldots, x_{n}\right)}{Z(B,-M)} \prod_{i=1}^{n} M\left(\mathrm{~d} x_{i}\right) .
$$

The normalising factor $Z(B,-M)$ is the partition function of the hard-sphere model. The argument $-M$ cancels the alternating sign in the definition of $Z$. For diffuse $M, h_{B, M}$ equals a Poisson $(M) \mathrm{PP}$ on $B$ conditioned to be 1-hard-core.

The analysis of the hard-sphere model centres on the partition function and derived quantities, in particular ratios (reduced correlations) and its logarithm (free energy). Lower bounds on $Z$ and $z$ and their logarithms at negative fugacity play a key role in the low fugacity case (the high temperature case) and establish uniqueness of the Gibbs measure [17, 32. A well-known tool is the cluster expansion, a series expansion of $\log Z$ [28. It fails first at negative fugacities, that is along the boundary of $\mathcal{M}^{>}$. Details are in Section 4.2

\subsection{Sufficient conditions for exponential bounds}

This section contains several LLLs in Theorems 8 and 10 and Corollary 11. Theorem 9 discusses the relation between $\mathcal{M}^{>}$and $\mathcal{M}^{\text {sh }}$. The proofs are in Section 6 
Let $\mathcal{B}^{1}$ be the set of Borel sets of diameter less than one. Let $\sqcup$ denote disjoint union. The unit partition number of a Borel set $B \in \mathcal{B}$ is

$$
\kappa(B):=\inf \left\{k \in \mathbb{N} \mid \exists A_{1}, \ldots, A_{k} \in \mathcal{B}^{1}: B=\bigsqcup_{i=1}^{k} A_{i}\right\} .
$$

Let $U(x)$ be the open unit sphere around $x$. The nice case are spaces where the unit-scale structure has an uniform exponential growth bound on spheres of radius $r$. Spaces such as $\mathbb{R}^{d}$, the hyperbolic plane or graphs with uniformly bounded degree fulfil this, but infinite dimensional metric spaces such as $l_{2}$ do not. This is equivalent to the bound

$$
K:=\sup \{\kappa(U(x)) \mid x \in \mathcal{X}\}<\infty .
$$

The first sufficient condition generalises the symmetric $L L L 16$ and Dobrushin's condition [14] respectively.

Theorem 8. Let $M \in \mathcal{M}^{b}$. If (1) holds and, for each $A \in \mathcal{B}^{1}$,

$$
M(A) \leq \frac{(K+1)^{K+1}}{(K+2)^{K+2}}
$$

then $M \in \mathcal{M}^{>}$and, for all $A, B \in \mathcal{B}^{b}$,

$$
z(A, B) \geq\left(\frac{K+1}{K+2}\right)^{\kappa(A \backslash B)}>0 .
$$

Under condition (1), a slight loss of precision sharpens the positive lower bound in the positive phase to exponential lower bounds. Proposition 9 shows that $\mathcal{M}^{\text {sh }}$ is a subset of the closure of $\mathcal{M}^{>}$. In general, $\mathcal{M}^{\text {sh }}$ is not the closure of $\mathcal{M}^{>}$[32, Chapter 8]. It is so on a finite graph, but already fails on a connected infinite graph.

Theorem 9. Let $\alpha>0$. If $(1+\alpha) M \in \mathcal{M}^{\text {sh }}$ and (1) holds, then $M \in \mathcal{M}^{>}$and, for all $A, B \in \mathcal{B}^{b}, z(A, B) \geq\left(\frac{\alpha}{1+\alpha}\right)^{\kappa(A \backslash B)}>0$.

The second sufficient condition generalises the asymmetric $L L L$ [16]. It resembles a continuous version of the Kotecky-Preiss condition [24].

Theorem 10. Let $M, N \in \mathcal{M}^{b}$ with $N$ being absolutely continuous with respect to $M$. If, for each $A \in \mathcal{B}^{1}$,

$$
\int_{A} \exp (N(U(x) \backslash A)) M(\mathrm{~d} x) \leq 1-\exp (-N(A)),
$$

then $M \in \mathcal{M}^{>}$and, for all $A, B \in \mathcal{B}^{b}$,

$$
z(A, B) \geq \exp (-N(A \backslash B))>0 .
$$

A stronger and more practical version of condition $3 \mathrm{a}$ is

$$
\forall A \in \mathcal{B}^{1}, M \text {-a.e. } x \in A: \quad M(A) \exp (N(U(x) \backslash A)) \leq 1-\exp (-N(A)) .
$$

Specialising (4) to the space $\mathbb{R}^{d}$ with the Lebesgue measure yields an explicit bound. 


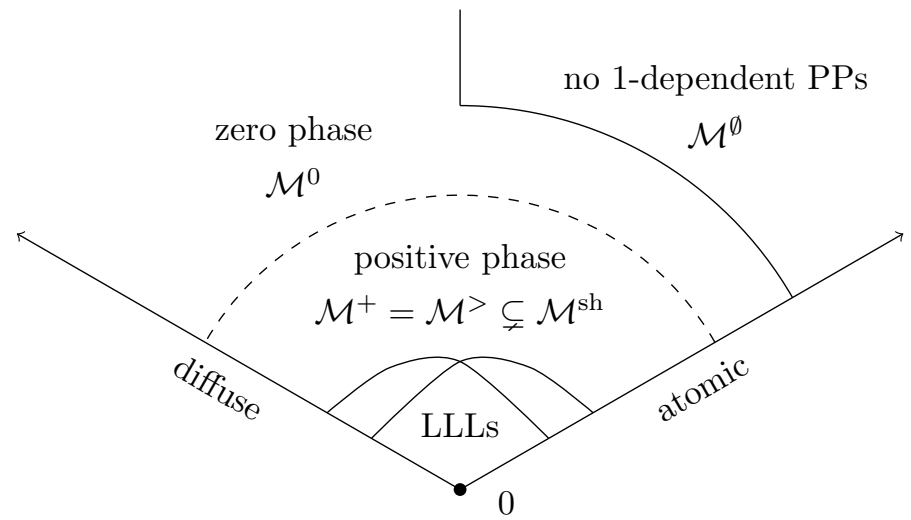

Figure 1: The cone of boundedly finite Borel measures $\mathcal{M}^{b}$, with diffuse measures on the left axis, atomic measures on the right axis and the zero measure in the apex. The positive and zero phases $\left(\mathcal{M}^{+}\right.$and $\left.\mathcal{M}^{0}\right)$ and $\mathcal{M}^{\emptyset}$ partition the cone. The sets $\mathcal{M}^{+}, \mathcal{M}^{\text {sh }}$ and $\mathcal{M}^{>}$are down-sets, whereas $\mathcal{M}^{\emptyset}$ and $\mathcal{M}^{0} \sqcup \mathcal{M}^{\emptyset}$ are up-sets. The dotted line represents the atypical closure properties of $\mathcal{M}^{>}$ and contains $\mathcal{M}^{\text {sh }} \backslash \mathcal{M}^{>}$. The LLLs carve out parts of the positive phase and guarantee exponential lower bounds.

Corollary 11. Consider $\mathcal{X}=\mathbb{R}^{d}$ with the Lebesgue measure $\mathcal{L}$. Let $V$ be the volume of the $d$-dimensional unit sphere. For $\lambda \leq 1 /(e V)$, let $\alpha$ be the unique solution of $\lambda=\alpha \exp (-\alpha V)$ in $[0,1 /(\mathrm{eV})]$. This implies that $\lambda \mathcal{L} \in \mathcal{M}^{>}$and, for all $A, B \in \mathcal{B}^{b}, z(A, B) \geq \exp (-\alpha \mathcal{L}(A \backslash B))>0$.

In the context of the hard-sphere model, Corollary 11 yields a uniform upper bound on finite volume free energies. That is, $\sup \left\{-\frac{\log Z(B,-\lambda \mathcal{L})}{\mathcal{L}(B)} \mid B \in \mathcal{B}^{b}\right\} \leq \alpha$. Together with taking the limit of $-\frac{\log Z(B,-\lambda \mathcal{L})}{\mathcal{L}(B)}$ in the van Hove sense [31, Definition 2.1.1], this implies the existence and complete analyticity of the infinite volume free energy for fugacities less than $1 /(\mathrm{eV})$. Thus, Corollary 11 gives an alternative proof, avoiding cluster expansion, of a classic result for uniqueness of the Gibbs measure of the hard-sphere model on $\mathbb{R}^{d}$ for small fugacities 31 , (5.2) in Section 4.5].

The LLLs and derived bounds here are not optimal. For example, the optimal bound on $\mathbb{R}$ in the context of Corollary 11 is $1 / e$ instead of $1 /(2 e)$ [19. There are two reasons to not pursue further improvements of the LLLs here. First, improvements in the main cases of interest are already present in the literature on the hard-core and hard-sphere models [17, 18. Second, in the context of Corollary 11, the best bounds differ by at most a factor of $e$ 31, (5.17) in Section 4.5].

\subsection{Stochastic domination and order}

A PP law $\varphi$ stochastically dominates a PP law $\xi$, if there is a coupling of them such that $\varphi$ contains almost surely all of $\xi$ 's points. Stochastic domination implies that $\varphi$ 's avoidance function is smaller than $\xi$ 's avoidance function. In the context of 1-dependent BRFs on locally finite graphs, the existence of Shearer's 
$\mathrm{PP}$ is equivalent to uniform stochastic domination by a Bernoulli product field [25. 37]. Uniform exponential lower bounds from the LLLs are a first step towards extending the stochastic domination result to 1-dependent PPs.

Stochastic domination of Shearer's PP by a Poisson PP would permit a probabilistic construction and simulation by thinning the dominating Poisson PP. The intrinsic coupling of Shearer's PP by independent thinning implies that stochastic domination for the largest intensities in $\mathcal{M}^{\text {sh }}$ suffices [30].

Using the terminology from [9], $\eta_{M}$ is weakly sub Poisson. This means that its moments and avoidance function are both smaller than the ones of a Poisson $(M)$ PP. The first follows from Proposition 28 and (7). The second follows by applying [9, Prop 3.1] to the implication of Proposition 19 , that, for disjoint $A, B \in \mathcal{B}^{b}$,

$$
\mathbb{P}\left(\eta_{M}(A \sqcup B)=0\right)=Z(A \sqcup B, M) \leq \mathbb{P}\left(\eta_{M}(A)=0\right) \mathbb{P}\left(\eta_{M}(B)=0\right) .
$$

Being weakly-sub Poisson yields concentration inequalities [5, Sec 3.3].

\section{About one-dependent PPs}

This section discusses different notions of 1-dependence in (5). Proposition 14 presents a key inequality of avoidance functions of 1-dependent PPs. Proposition 12 characterises the avoidance functions of 1-dependent PPs. Proposition 15 investigates the existence of simple 1-dependent PPs.

For $A \in \mathcal{B}$ and a $\mathrm{PP} \xi$, let $A \xi$ be the restriction of $\xi$ to $A$. Recall that the metric is $\delta$. $\mathrm{APP} \xi$ is strong 1-dependent, if, for all $A, B \in \mathcal{B}$,

$$
\delta(A, B):=\inf \{\delta(x, y) \mid x \in A, y \in B\} \geq 1 \Rightarrow A \xi \text { is independent of } B \xi \text {. }
$$

All the examples in the introduction, the $\mathrm{PP}$ in the zero phase of Theorem 1 . the PP counterexample in the proof of Theorem 6. Shearer's PP and the PP in Proposition 15 are strong 1-dependent.

Proposition 12. A PP is strong 1-dependent, if and only if its avoidance function $Q$ is 1-multiplicative. That is, for all $A, B \in \mathcal{B}, \delta(A, B) \geq 1 \Rightarrow Q(A \cup B)=$ $Q(A) Q(B)$.

Proof. The necessity is evident, and the sufficiency follows from the fact that the avoidance function determines the law of a simple PP [11, 9.2.XIII].

A PP $\xi$ is weak 1 -dependent, if, for $M$-a.e. $x$ and every $B \in \mathcal{B}$,

$$
\delta(x, B):=\delta(\{x\}, B) \geq 1 \Rightarrow \mathbb{P}_{x}(\xi(B)=0)=\mathbb{P}(\xi(B)=0) .
$$

In other words, weak 1-dependence reduces Palm probabilities for events at distance more than one away from the base point to normal probabilities.

Proposition 13. A strong 1-dependent PP is weak 1-dependent.

Examples of weak, but not strong, 1-dependent PPs are mixtures of random shifts of strong 1-dependent BRFs in [25, Section 5].

Proof. Let $\xi \in \mathcal{C}(M)$. The Campbell measure $C$ [11, (13.1.1a)] on $(B, E) \in$ $\mathcal{B} \times \sigma(\xi)$ is $C(B \times E):=\sum_{n=1}^{\infty} n \mathbb{P}(\xi(B)=n, \xi \in E)$. If $A, B \in \mathcal{B}^{b}$ with 
$\delta(A, B) \geq 1$ and $E \in \sigma(A \xi)$ with embedding $E^{\prime}$ into $\sigma(\xi)$, then strong 1dependence (5a) allows to factorise $C\left(B \times E^{\prime}\right)=\sum_{n=1}^{\infty} n \mathbb{P}(\xi(B)=n, A \xi \in$ $E)=\mathbb{E}(\xi(B)) \mathbb{P}(A \xi \in E)$. Hence, $M$-a.e., the Palm density on $\sigma(A \xi)$ simplifies to $\mathbb{P}_{x}(\xi \in E)=\frac{d C(. \times E)}{d \mathbb{E}(\xi(\{.\}))}=\mathbb{P}(\xi \in E)$. As the $\sigma$-algebras of the form $\sigma(A \xi)$, for $A \in \mathcal{B}^{b}$ with $A \cap U(x)=\emptyset$, generate $\sigma((X \backslash U(x)) \xi)$, weak 1-dependence follows.

The inequality in Proposition 14 lies at the core of the dichotomy in Theorem 1 and the minimality of the avoidance function of Shearer's PP in Theorem 4. Proposition 17 shows that the 1-hard-core of Shearer's PP makes it the only 1-dependent PP to fulfil the inequality uniformly as an equality. The inequality motivates the relaxation from strong to weak 1-dependence. Thus, $\mathcal{C}(M)$ may be extended to be the class of weak 1-dependent simple PPs with intensity measure $M$.

Proposition 14. For all $\xi \in \mathcal{C}(M), A \in \mathcal{B}^{1}$ and $B \in \mathcal{B}^{b}$ with $\mathbb{P}(\xi(B)=0)>0$,

$$
\frac{\mathbb{P}(\xi(A \cup B)=0)}{\mathbb{P}(\xi(B)=0)} \geq 1-\int_{A \backslash B} \frac{\mathbb{P}(\xi(B \backslash U(x))=0)}{\mathbb{P}(\xi(B)=0)} M(\mathrm{~d} x) .
$$

Proof. With $C$ the Campbell measure and the event $E:=\{\omega \mid \xi(B)=0\}$,

$$
\begin{aligned}
\mathbb{P}(\xi(A \backslash B) \geq 1, \xi(B)=0) & \leq \sum_{n=1}^{\infty} n \mathbb{P}(\xi(A \backslash B)=n, \xi \in E) \\
& =C((A \backslash B) \times E) \\
& =\int_{A \backslash B} \mathbb{P}_{x}(\xi(B)=0) M(\mathrm{~d} x) \\
& \leq \int_{A \backslash B} \mathbb{P}_{x}(\xi(B \backslash U(x))=0) M(\mathrm{~d} x) \\
& \int_{A \backslash B} \mathbb{P}(\xi(B \backslash U(x))=0) M(\mathrm{~d} x)
\end{aligned}
$$

and

$$
\begin{aligned}
\mathbb{P}(\xi(A \cup B)=0) & =\mathbb{P}(\xi(B)=0)-\mathbb{P}(\xi(A \backslash B) \geq 1, \xi(B)=0) \\
& \geq \mathbb{P}(\xi(B)=0)-\int_{A \backslash B} \mathbb{P}(\xi(B \backslash U(x))=0) M(\mathrm{~d} x) .
\end{aligned}
$$

Proposition 15. Let $M \in \mathcal{M}^{b}$. If $M$ has no atom of mass greater than one, then there exists a strong 0-dependent PP with intensity measure $M$. If $M$ has an atom of mass greater than one, then no simple PP with intensity measure $M$ exists.

Proof. For each $\xi \in \mathcal{C}(M)$ and atom $x$ of $M, 1 \geq \mathbb{E}(\xi(\{x\}))=M(\{x\})=: m_{x}$. Hence, an atom of mass greater than one contradicts simpleness of the PP.

For the converse, let $M \in \mathcal{M}^{b}$ without atoms greater than 1 . Let $\mathcal{A}$ and $\mathcal{D}$ be the atomic and diffuse support domains of $M$ respectively. Let $\mathcal{A}_{=}$and $\mathcal{A}_{<}$be the locations of atoms of mass equal to or less than one respectively. Construct a measure $N$ with the same atomic and diffuse domains as follows. On $\mathcal{D}$, let 
$N \cap \mathcal{D}:=M \cap \mathcal{D}$. On $\mathcal{A}$, let $n_{x}:=N(\{x\}):=-\log \left(1-m_{x}\right)$, if $x \in \mathcal{A}_{<}$, and 0 else. For $B \in \mathcal{B}^{b}$, let $m_{B}:=\max \left\{m_{x} \mid x \in B \cap \mathcal{A}_{<}\right\}$. As $M(B)<\infty$, so is $m_{B}<1$. Also, $N(B \backslash \mathcal{A})=M(B \backslash \mathcal{A})<\infty$. The inequality

$$
\forall y \in\left[0,1\left[: \quad-\log (1-y)=\sum_{n=1}^{\infty} \frac{y^{n}}{n} \leq \sum_{n=1}^{\infty} y^{n}=\frac{y}{1-y}\right.\right.
$$

entails the bounded finiteness of $N$ on $\mathcal{A}$ via

$$
N(B \cap \mathcal{A})=-\sum_{x \in B \cap \mathcal{A}_{<}} \log \left(1-m_{x}\right) \stackrel{6}{\leq} \sum_{x \in B \cap \mathcal{A}_{<}} \frac{m_{x}}{1-m_{x}} \leq \frac{M\left(B \cap \mathcal{A}_{<}\right)}{1-m_{B}}<\infty .
$$

The aim is to construct a strong 0-dependent $\mathrm{PP}$ with intensity $M$. Let $\varphi$ be the Poisson PP with intensity $N$. It may not be simple because of atoms in $N$. Let $\varphi^{\bullet}$ be its simple support PP, collapsing multiple points of $\varphi$. Let $\xi:=\varphi^{\bullet}+\sum_{x \in \mathcal{A}_{-}} \delta_{x}$. The 0 -dependence of $\xi$ holds by construction and it remains to verify that $\xi \overline{\bar{h}}$ has intensity $M$. On the diffuse part of $M, \varphi$ is simple, whence the intensity of $\xi$ is $M$. On $\mathcal{A}_{=}, \varphi$ is almost-surely zero, but the atoms of mass one are present deterministically in $\xi$. For all $x \in \mathcal{A}_{<}$,

$$
\mathbb{E}(\xi(\{x\}))=\mathbb{P}(\xi(\{x\})=1)=1-\mathbb{P}(\varphi(\{x\})=0)=1-e^{-n_{x}}=m_{x} .
$$

Hence, for each $B \subseteq \mathcal{B}^{b}$, the intensity measure is

$$
\mathbb{E}\left(\xi\left(B \cap \mathcal{A}_{<}\right)\right)=\sum_{x \in B \cap \mathcal{A}_{<}} m_{x}=M\left(B \cap \mathcal{A}_{<}\right) .
$$

\section{Properties of the generating function}

Most properties of $Z$ and $z$ are trivial, if one knows that $Z$ is the avoidance function of Shearer's PP. The properties are needed to establish the existence of Shearer's PP first, though. The equality in Proposition 17 (cf. the inequality in Proposition 14) and monotonicity in Proposition 19 are the most important ones.

Let $[n]:=\{1, \ldots, n\}$, with $[0]:=\emptyset$. For a set $S$, let $S^{n}$ be the Cartesian product of $n$ copies of $S$, with $S^{0}:=\emptyset$. Empty products evaluate to 1 and empty sums to 0 . For $B \in \mathcal{B}^{b}, M \in \mathcal{M}^{b}$ and $\lambda \in[0, \infty[$, let $\lambda M$ be the scaling of $M$ by the factor $\lambda$ and $\left.M\right|_{B}$ the restriction of $M$ to $B$.

\subsection{Basic properties}

For $B \in \mathcal{B}^{b}$ and $M \in \mathcal{M}^{b}$, a basic bound is

$$
\int_{B^{n}} h_{n}\left(x_{1}, \ldots, x_{n}\right) \prod_{i=1}^{n} M\left(\mathrm{~d} x_{i}\right) \leq M(B)^{n} .
$$

Also, for each $B \in \mathcal{B}^{\kappa}, n \in \mathbb{N}_{0}$ and $x_{1}, \ldots, x_{n} \in B$,

$$
h_{n}\left(x_{1}, \ldots, x_{n}\right)=1 \Leftrightarrow n \leq \kappa(B) .
$$

Proposition 16. $Z$ is well-defined and 1-multiplicative as in Proposition 12 . 
Proof. For $k \in \mathbb{N}_{0}$, the bound (7) implies that

$$
\left|\sum_{n=k}^{\infty} \frac{(-1)^{n}}{n !} \int_{B^{n}} h_{n}\left(x_{1}, \ldots, x_{n}\right) \prod_{i=1}^{n} M\left(\mathrm{~d} x_{i}\right)\right| \leq \sum_{n=k}^{\infty} \frac{M(B)^{n}}{n !} .
$$

For $k=0$, this yields $|Z(B)| \leq \exp (M(B))$. For $k \rightarrow \infty$, this shows the convergence of the series $Z(B)$.

Let $A, B \in \mathcal{B}^{b}$ with $\delta(A, B) \geq 1$. The 1-hard-core condition trivially holds for pairs in $A \times B$. For $n, m \in \mathbb{N}_{0}, x_{1}, \ldots, x_{n} \in A$ and $y_{1}, \ldots, y_{m} \in B$, $h_{n+m}\left(x_{1}, \ldots, x_{n}, y_{1}, \ldots, y_{m}\right)=h_{n}\left(x_{1}, \ldots, x_{n}\right) h_{m}\left(y_{1}, \ldots, y_{m}\right)$. Hence, 1-multiplicativity follows from

$$
\begin{aligned}
Z(A \cup B)= & \sum_{n=0}^{\infty} \frac{1}{n !} \int_{(A \cup B)^{n}}(-1)^{n} h_{n}\left(x_{1}, \ldots, x_{n}\right) \prod_{i=1}^{n} M\left(\mathrm{~d} x_{i}\right) \\
= & \sum_{n=0}^{\infty} \frac{1}{n !} \sum_{j=0}^{n}\left(\begin{array}{l}
n \\
j
\end{array}\right)\left(\int_{A^{j}}(-1)^{j} h_{j}\left(x_{1}, \ldots, x_{j}\right) \prod_{i=1}^{j} M\left(\mathrm{~d} x_{i}\right)\right) \\
& \times\left(\int_{B^{n-j}}(-1)^{n-j} h_{n-j}\left(x_{1}, \ldots, x_{n-j}\right) \prod_{i=1}^{n-j} M\left(\mathrm{~d} x_{i}\right)\right) \\
= & \left(\sum_{n=0}^{\infty} \frac{1}{n !} \int_{A^{n}}(-1)^{n} h_{n}\left(x_{1}, \ldots, x_{n}\right) \prod_{i=1}^{n} M\left(\mathrm{~d} x_{i}\right)\right) \\
& \times\left(\sum_{n=0}^{\infty} \frac{1}{n !} \int_{B^{n}}(-1)^{n} h_{n}\left(x_{1}, \ldots, x_{n}\right) \prod_{i=1}^{n} M\left(\mathrm{~d} x_{i}\right)\right) \\
= & Z(A) Z(B) .
\end{aligned}
$$

Proposition 17. For all $A \in \mathcal{B}^{1}$ and $B \in \mathcal{B}^{b}$ with $Z(B)>0$,

$$
z(A, B)=1-\int_{A \backslash B} z(B \cap U(x), B \backslash U(x))^{-1} M(\mathrm{~d} x) .
$$

Proof. By (8), at most one point of an 1-hard-core configuration $C$ lies in $A$. If $y \in A \cap C$, then the 1-hard-core implies that $C \cap(B \backslash U(y))=\emptyset$. This leads to

$$
\begin{aligned}
Z(B)= & \sum_{n=0}^{\infty} \frac{(-1)^{n}}{n !} \int_{B^{n}} h_{n}\left(x_{1}, \ldots, x_{n}\right) \prod_{i=1}^{n} M\left(\mathrm{~d} x_{i}\right) \\
= & \sum_{n=0}^{\infty} \frac{(-1)^{n}}{n !}\left(\int_{(B \backslash A)^{n}} h_{n}\left(x_{1}, \ldots, x_{n}\right) \prod_{i=1}^{n} M\left(\mathrm{~d} x_{i}\right)\right. \\
& \left.+n \int_{A \times B^{n-1}} h_{n}\left(x_{1}, \ldots, x_{n}\right) \prod_{i=1}^{n} M\left(\mathrm{~d} x_{i}\right)\right)
\end{aligned}
$$

A point in $A$ excludes the possibility of other points in $A$. Thus,

$$
\begin{aligned}
Z(B)= & \sum_{n=0}^{\infty} \frac{(-1)^{n}}{n !} \int_{(B \backslash A)^{n}} h_{n}\left(x_{1}, \ldots, x_{n}\right) \prod_{i=1}^{n} M\left(\mathrm{~d} x_{i}\right) \\
& -\int_{A} \sum_{n=1}^{\infty} \frac{(-1)^{n-1}}{(n-1) !} \int_{B^{n-1}} h_{n}\left(x_{1}, \ldots, x_{n-1}, y\right) \prod_{i=1}^{n-1} M\left(\mathrm{~d} x_{i}\right) M(\mathrm{~d} y)
\end{aligned}
$$




$$
\begin{aligned}
& =Z(B \backslash A)-\int_{A} \sum_{n=0}^{\infty} \frac{(-1)^{n}}{n !} \int_{(B \backslash U(y))^{n}} h_{n}\left(x_{1}, \ldots, x_{n}\right) \prod_{i=1}^{n} M\left(\mathrm{~d} x_{i}\right) M(\mathrm{~d} y) \\
& =Z(B \backslash A)-\int_{A} Z(B \backslash U(y)) M(\mathrm{~d} y) .
\end{aligned}
$$

\subsection{Cluster expansion and monotonicity}

Cluster expansion is a series expansion of the logarithm of a generating series. It is a classic technique from statistical mechanics [31, Section 4.4].

Proposition 18. Let $A, B \in \mathcal{B}^{\kappa}$ and $M \in \mathcal{M}^{b}$ with $\left.M\right|_{\mathcal{X} \backslash(A \cup B)}=0$, i.e., it is concentrated on $A \cup B$. The statement $\forall N \leq M: N \in \mathcal{M}^{>}$holds, if and only if

$$
\log z(A, B, M)=-\sum_{n=1}^{\infty} \frac{1}{n !} \int_{(A \cup B)^{n} \backslash B^{n}} \mathbf{P}\left(x_{1}, \ldots, x_{n}\right) \prod_{i=1}^{n} M\left(\mathrm{~d} x_{i}\right)
$$

is a convergent series, with $\mathbf{P}\left(x_{1}, \ldots, x_{n}\right) \in \mathbb{N}_{0}$ well-defined.

Proof. A cluster expansion of the partition function of a hard-sphere gas with radius one at negative fugacity [28] with an application of Penrose's identity [29] implies that the coefficients $\mathbf{P}\left(x_{1}, \ldots, x_{n}\right)$ have the desired properties.

Some background about the coefficients $\mathbf{P}\left(x_{1}, \ldots, x_{n}\right)$. Create a graph $G$ with vertices $[n]$ and edges $(i, j) \in G$, if $\delta\left(x_{i}, x_{j}\right)<1$. If $G$ is connected, then regard the term

$$
\sum_{H \text { spans } G}(-1)^{|E(H)|} .
$$

Penrose shows that this counts the cardinality of a subset of the spanning trees of $G$, modulo a sign. Let $\mathbf{P}\left(x_{1}, \ldots, x_{n}\right)$ be this count, if $G$ is connected, and 0 otherwise. This work only uses the non-negativity and finiteness of $\mathbf{P}\left(x_{1}, \ldots, x_{n}\right)$.

Proposition 19. As long as they are positive, the functions $Z$ and $z$ are monotone decreasing in both space and measure.

Proof. As $-\log z(A, B, M)$ is a sum over integrals over non-negative integrands, it is monotone increasing in both the integration domains and the measure. Because $Z(\emptyset)=1$, the same holds for the cluster expansion of $Z$.

Monotonicity implies the following telescoping identity. For all $A, B \in \mathcal{B}^{b}$ with $Z(B)>0$ and every partition $\left\{A_{i}\right\}_{i=1}^{n}$ of $A \backslash B$ by elements of $\mathcal{B}^{b}$,

$$
z(A, B)=\prod_{i=1}^{n} z\left(A_{i}, B \sqcup \bigsqcup_{j=1}^{i-1} A_{j}\right)
$$

\subsection{Continuity properties}

This section investigates continuity properties of $Z$ in both space and measure.

Proposition 20. For $B \in \mathcal{B}^{b}$ and $M \in \mathcal{M}^{b}$, the function $f_{B}:[0, \infty[\rightarrow \mathbb{R}, \lambda \mapsto$ $Z(B, \lambda M)$ is continuous. 
Proof. The scaling of $M$ by $\lambda$ implies that continuity of $f_{B}$ at 1 suffices. This follows from the bound, for each $\varepsilon$ with $|\varepsilon|<1$,

$$
\begin{aligned}
& |Z(B,(1+\varepsilon) M)-Z(B, M)| \\
= & \left|\sum_{n=0}^{\infty} \frac{(-1)^{n}}{n !} \int_{B^{n}} h_{n}\left(x_{1}, \ldots, x_{n}\right)\left(1-(1-\varepsilon)^{n}\right) \prod_{i=1}^{n} M\left(\mathrm{~d} x_{i}\right)\right| \\
\leq & \sum_{n=1}^{\infty} \frac{1}{n !} \sum_{j=1}^{n}\left(\begin{array}{c}
n \\
j
\end{array}\right)|\varepsilon|^{j} \int_{B^{n}} \prod_{i=1}^{n} M\left(\mathrm{~d} x_{i}\right) \\
\underline{\text { 7 }} & \sum_{n=0}^{\infty} \frac{1}{n !} 2^{n}|\varepsilon| M(B)^{n}=|\varepsilon| \exp (2 M(B)) .
\end{aligned}
$$

Proposition 21. For $B \in \mathcal{B}^{b}$, let $\lambda_{B}:=\inf \left\{\lambda \mid f_{B}(\lambda)<0\right\}$. If $B \supseteq A \in \mathcal{B}^{b}$, then $\lambda_{B} \leq \lambda_{A}$. If $M(B)>0$, then $\left.\lambda_{B}=\min \left\{\lambda \mid f_{B}(\lambda)=0\right\} \in\right] 0, \infty[$ and $\left.\lambda_{B} M\right|_{B} \in \mathcal{M}^{\text {sh }}$.

Proof. First prove the contra-variance of $\lambda_{B}$. Let $\Lambda<\lambda_{B}$. Proposition 18 implies that $f_{B}(\lambda) \leq f_{A}(\lambda)$, for all $\lambda \leq \Lambda$. Hence, $\lambda_{A} \geq \lambda_{B}$.

If $M(B)>0$, then there is $B \supseteq A \in \mathcal{B}^{1}$ with $M(A)>0$. Because $f_{A}(\lambda)=1-$ $\lambda M(A), \lambda_{A}=1 / M(A)$. Contra-variance yields $\lambda_{B} \leq \lambda_{A}=1 / M(A)<\infty$. The continuity of $f_{B}$ from Proposition 20 implies together with $f_{B}(0)=Z(\emptyset)=1$ that $\lambda_{B}>0$.

The continuity of $f_{B}$ renders the infimum a minimum. Contra-variance implies that for all $\lambda \leq \lambda_{B}$ and $B \supseteq A \in \mathcal{B}^{b}, f_{A}(\lambda) \geq 0$. Proposition 18 implies that $\left.\lambda_{B} M\right|_{B} \in \mathcal{M}^{\text {sh }}$.

Proposition 22. Let $B \in \mathcal{B}^{b}$ be a continuity set of $M$, i.e., $M(\bar{B} \backslash B)=0$, where $\bar{B}$ is the closure of $B$. For each sequence $\left(B_{n}\right)_{n \in \mathbb{N}}$ in $\mathcal{B}^{b}$ decreasing to $B$ with $M\left(B_{1}\right)>0$, the limit $Z\left(B_{n}\right) \underset{n \rightarrow \infty}{\longrightarrow} Z(B)$ holds.

Proof. For $A, B \in \mathcal{B}^{b}$ with $A \subseteq B$ and $M(B)>0$, bound the difference as

$$
\begin{aligned}
|Z(B)-Z(A)| & =\left|\sum_{n=0}^{\infty} \frac{(-1)^{n}}{n !} \int_{B^{n} \backslash A^{n}} h_{n}\left(x_{1}, \ldots, x_{n}\right) \prod_{i=1}^{n} M\left(\mathrm{~d} x_{i}\right)\right| \\
& \leq \sum_{n=1}^{\infty} \frac{1}{n !}\left(M(B)^{n}-M(A)^{n}\right) \\
& =\sum_{n=1}^{\infty} \frac{1}{n !} \sum_{j=1}^{n}\left(\begin{array}{c}
n \\
j
\end{array}\right) M(B \backslash A)^{j} M(A)^{n-j} \\
& \leq \sum_{n=1}^{\infty} \frac{1}{n !} M(B \backslash A) 2^{n} \max \{1, M(B)\}^{n-1} \\
& \leq 2 M(B \backslash A) \exp (\max \{1, M(B)\}) .
\end{aligned}
$$

Thus, $\left|Z\left(B_{n}\right)-Z(B)\right| \leq 2 M\left(B_{n} \backslash B\right) \exp \left(\max \left\{1, M\left(B_{1}\right)\right\}\right) \frac{\text { continuity of } M \text { at } B}{n \rightarrow \infty}$ 0 . 


\subsection{Behaviour under the set difference operator}

The set difference operator $\Delta$ at $A \in \mathcal{B}$ transforms a set function $\phi: \mathcal{B} \rightarrow \mathbb{R}$ into $\Delta(A) \phi: \mathcal{B} \rightarrow \mathbb{R} \quad B \mapsto \phi(B)-\phi(B \cup A)$.

Lemma 23. For all $n \in \mathbb{N}$ and $A_{1}, \ldots, A_{n}, B \in \mathcal{B}$ and $\phi: \mathcal{B} \rightarrow \mathbb{R}$, iterated application of difference operators commutes and has the canonical form

$$
\Delta\left(\left\{A_{1}, \ldots, A_{n}\right\}\right):=\Delta\left(A_{1}\right)\left(\ldots\left(\Delta\left(A_{n}\right) \phi\right) \ldots\right)(B)=\sum_{I \subseteq[n]}(-1)^{|I|} \phi\left(B \cup \bigcup_{i \in I} A_{i}\right) .
$$

In particular, $\Delta(\{A\})=\Delta(A)$ and $\Delta(\emptyset)$ is the identity.

Proof. Commutativity follows from the canonical form for $n=2$. For $n=1$,

$$
\Delta(\{A\}) \phi(B)=\Delta(A) \phi(B)=\phi(B)-\phi(B \cup A) .
$$

Proceed by induction over $n$. The induction step from $n$ to $n+1$ is

$$
\begin{aligned}
& \Delta\left(\left\{A_{1}, \ldots, A_{n+1}\right\}\right) \phi(B) \\
= & \Delta\left(A_{n+1}\right)\left(\Delta\left(\left\{A_{1}, \ldots, A_{n}\right\}\right) \phi\right)(B) \\
= & \left(\Delta\left(\left\{A_{1}, \ldots, A_{n}\right\}\right) \phi\right)(B)-\left(\Delta\left(\left\{A_{1}, \ldots, A_{n}\right\}\right) \phi\right)\left(B \cup A_{n+1}\right) \\
= & \sum_{I \subseteq[n]}(-1)^{|I|} \phi\left(B \cup \bigcup_{i \in I} A_{i}\right)-\phi\left(B \cup \bigcup_{i \in I} A_{i} \cup A_{n+1}\right) \\
= & \sum_{I \subseteq[n+1]}(-1)^{|I|} \phi\left(B \cup \bigcup_{i \in I} A_{i}\right) .
\end{aligned}
$$

Lemma 24. Let $\left\{A_{i}\right\}_{i=1}^{n}$ be disjoint Borel sets. For $I \subseteq[n]$, let $A_{I}:=\bigsqcup_{i \in I} A_{i}$. For each $\phi: \mathcal{B} \rightarrow \mathbb{R}$, one has $\sum_{I \subseteq[n]} \Delta\left(\left\{A_{i}\right\}_{i \in I}\right) \phi\left(A_{[n] \backslash I}\right)=\phi(\emptyset)$.

Proof. Abbreviate $\bar{\phi}(I):=\phi\left(A_{I}\right)$. As $A_{\emptyset}=\emptyset$, so is $\bar{\phi}(\emptyset)=\phi\left(A_{\emptyset}\right)=\phi(\emptyset)$.

$$
\begin{aligned}
\sum_{I \subseteq[n]} \Delta\left(\left\{A_{i}\right\}_{i \in I}\right) \bar{\phi}([n] \backslash I) & =\sum_{I \subseteq[n]} \sum_{J \subseteq I}(-1)^{|J|} \bar{\phi}(([n] \backslash I) \sqcup J) \\
& =\sum_{I \subseteq[n]} \sum_{J \subseteq I}(-1)^{|J|+|I|} \bar{\phi}([n] \backslash J) \\
& =\sum_{J \subseteq[n]}(-1)^{|J|} \bar{\phi}([n] \backslash J) \sum_{J \subseteq I \subseteq[n]}(-1)^{|I|} \\
& =\sum_{J=[n]}(-1)^{|J|} \bar{\phi}([n] \backslash J)(-1)^{|J|} \\
& =\bar{\phi}(\emptyset)
\end{aligned}
$$

Proposition 25. If $M \in \mathcal{M}^{\text {sh }}$, then $Z$ is completely monotone, i.e., for each $n \in \mathbb{N}_{0}$ and all $A_{1}, \ldots, A_{n}, B \in \mathcal{B}^{\kappa}$, the iterated difference $\Delta\left(\left\{A_{i}\right\}_{i=1}^{n}\right) Z(B)$ is non-negative.

Proof. Proceed by induction over $n$. For $n=0, M \in \mathcal{M}^{\text {sh }}$ implies that $\Delta(\emptyset) Z(B)=$ $Z(B) \geq 0$. For $n=1$, the monotonicity in space of $Z$ from Proposition 19 implies that $\Delta\left(\left\{A_{1}\right\}\right) Z(B)=\Delta\left(A_{1}\right) Z(B)=Z(B)-Z(B \cup A) \geq 0$. The induction 
step from $n$ to $n+1$ needs more preparation. For $I \subseteq[n]$, let $A_{I}:=\bigcup_{i \in I} A_{i}$. For $x \in A_{n+1}$, let $B^{x}:=B \backslash U(x)$ and, for $i \in[n+1]$, let $A_{i}^{x}:=A_{i} \backslash U(x)$. In particular, $A_{n+1}^{x}=A_{n+1} \backslash U(x)=\emptyset$. As $\Delta(\emptyset) \phi=\phi$, the degree of the iterated difference decreases in

$$
\begin{aligned}
& \Delta\left(\left\{A_{i}\right\}_{i=1}^{n+1}\right) Z(B) \\
& =\Delta\left(A_{n+1}\right)\left(\Delta\left(\left\{A_{i}\right\}_{i=1}^{n}\right) Z(B)\right) \\
& =\Delta\left(\left\{A_{i}\right\}_{i=1}^{n}\right) Z(B)-\Delta\left(\left\{A_{i}\right\}_{i=1}^{n}\right) Z\left(B \cup A_{n+1}\right) \\
& \text { 11] } \sum_{I \subseteq[n]}(-1)^{|I|} Z\left(B \cup A_{I}\right)-\sum_{I \subseteq[n]}(-1)^{|I|} Z\left(B \cup A_{I} \cup A_{n+1}\right) \\
& \stackrel{9]}{=} \sum_{I \subseteq[n]}(-1)^{|I|}(Z\left(B \cup A_{I}\right)-\underbrace{Z\left(B \cup A_{I} \cup A_{n+1}\right)}) \\
& =\sum_{I \subseteq[n]}(-1)^{|I|}(Z\left(B \cup A_{I}\right)-\overbrace{Z\left(B \cup A_{I}\right)+\int_{A_{n+1}} Z\left(\left(B \cup A_{I}\right) \backslash U(x)\right) M(\mathrm{~d} x)}) \\
& =\sum_{I \subseteq[n]}(-1)^{|I|} \int_{A_{n+1}} Z\left(B^{x} \cup A_{I}^{x}\right) M(\mathrm{~d} x) \\
& =\int_{A_{n+1}} \underbrace{\Delta\left(\left\{A_{i}^{x}\right\}_{i=1}^{n}\right) Z\left(B^{x}\right)}_{\geq 0 \text { by the induction hypothesis }} M(\mathrm{~d} x) \geq 0 .
\end{aligned}
$$

Proposition 26. Let $A_{1}, \ldots, A_{n}, B \in \mathcal{B}^{\kappa}$ be disjoint. If $\kappa\left(B \sqcup \bigsqcup_{i=1}^{n} A_{i}\right)<n$, then $\Delta\left(\left\{A_{i}\right\}_{i=1}^{n}\right) Z(B)=0$.

Proof. For $I \subseteq[n]$, let $A_{I}:=\bigsqcup_{i \in I} A_{i}$. Let $A:=A_{[n]}$.

$$
\begin{aligned}
& \Delta\left(\left\{A_{i}\right\}_{i=1}^{n}\right) Z(B) \\
= & \sum_{I \subseteq[n]}(-1)^{|I|} Z\left(B \sqcup A_{I}\right) \\
= & \sum_{I \subseteq[n]}(-1)^{|I|} \sum_{m=0}^{\infty} \frac{(-1)^{m}}{m !} \int_{\left(B \sqcup A_{I}\right)^{m}} h_{m}\left(x_{1}, \ldots, x_{m}\right) \prod_{i=1}^{m} M\left(\mathrm{~d} x_{i}\right)
\end{aligned}
$$

For $m \in \mathbb{N}_{0}$ and $x_{1}, \ldots, x_{m} \in A \sqcup B$, regard the indices $I\left(x_{1}, \ldots, x_{m}\right):=$ $\left\{i \in[n] \mid\left\{x_{1}, \ldots, x_{m}\right\} \cap A_{i} \neq \emptyset\right\}$ of the partition elements containing the points.

$$
\begin{aligned}
& =\sum_{m=0}^{\infty} \frac{(-1)^{m}}{m !} \int_{\left(B \sqcup A_{I}\right)^{m}} h_{m}\left(x_{1}, \ldots, x_{m}\right) \sum_{I\left(x_{1}, \ldots, x_{m}\right) \subseteq I \subseteq[n]}(-1)^{|I|} \prod_{i=1}^{m} M\left(\mathrm{~d} x_{i}\right) \\
& =\sum_{m=0}^{\infty} \frac{(-1)^{m}}{m !} \int_{\left(B \sqcup A_{I}\right)^{m}} h_{m}\left(x_{1}, \ldots, x_{m}\right) \sum_{I\left(x_{1}, \ldots, x_{m}\right)=[n]}(-1)^{n} \prod_{i=1}^{m} M\left(\mathrm{~d} x_{i}\right)
\end{aligned}
$$

Using (8) yields $n \leq m \leq \kappa(A \sqcup B)<n$ and a zero integrand.

Proposition 27. Let $\left\{A_{i}\right\}_{i=1}^{n}$ be disjoint elements of $\mathcal{B}^{1}$. For $I \in[n]$, let $A_{I}:=$ $\bigsqcup_{i \in I} A_{i}$. For all $r \geq \kappa\left(A_{[n]}\right), \sum_{I \subseteq[n],|I| \leq r} \Delta\left(\left\{A_{i}\right\}_{i \in I}\right) Z\left(A_{[n] \backslash I}\right)=1$ holds.

Proof. Fill up with Proposition 26 and evaluate the sum with Lemma 24. 


\section{Proofs around Shearer's point process}

Section 5.1 contains the uniqueness and characterisation in Proposition 28 and the existence in Proposition 29. Together they imply Theorem 3. Section 5.2 proves the minimality of Shearer's PP for the avoidance function in Theorem 4. Section 5.4 discusses intrinsic couplings between Shearer's PP at different intensities and the monotonicity properties of the sets of measures. Section 5.5 shows that Shearer's PP law differs from well known hard-core or 1-dependent PP laws and references probabilistic constructions. The notation of Section 4 applies.

Besides the $\sigma$-algebra of all Borel sets $\mathcal{B}$, there are also the algebras of bounded, $\kappa$-finite and less than unit-diameter Borel sets $\mathcal{B}^{b}, \mathcal{B}^{\kappa}$ and $\mathcal{B}^{1}$ respectively. $\mathcal{B}^{\kappa}$ is an algebra of $\mathcal{B}^{1}$. Both algebras $\mathcal{B}^{b}$ and $\mathcal{B}^{\kappa}$ generate the $\sigma$-algebra $\mathcal{B}$. The distinction between $\mathcal{B}^{\kappa}$ and $\mathcal{B}^{b}$ plays a key role, as key proofs employ induction over $\kappa$. The strategy is to first establish results on $\mathcal{B}^{\kappa}$, extend them by $\sigma$-finiteness to $\mathcal{B}$ and and project them onto $\mathcal{B}^{b}$. Sufficient conditions for $\mathcal{B}^{\kappa}$ and $\mathcal{B}^{b}$ to coincide are that $(\mathcal{X}, \delta)$ is either $\sigma$-compact or total (bounded sets are pre-compact). The structure of the space below the distance 1 plays no role.

\subsection{Characterisation, uniqueness and existence}

For $a, n \in \mathbb{N}_{0}$, let the falling factorial be $a^{[n]}:=\prod_{i \in[n]}(a-i+1)$. The factorial moment measure of a PP $\xi$ of order $n$ on $B \in \mathcal{B}$ is $\mathbb{E}\left(\xi(B)^{[n]}\right)$ [11, Section 9.5].

Proposition 28. If there exists a strong 1-dependent and 1-hard-core PP $\eta_{M}$ with intensity measure $M \in \mathcal{M}^{b}$, then its factorial moment measure of order $n$ at $B \in \mathcal{B}^{b}$ fulfils $\mathbb{E}\left(\eta_{M}(B)^{[n]}\right)=\int_{B^{n}} h_{n}\left(x_{1}, \ldots, x_{n}\right) \prod_{i=1}^{n} M\left(\mathrm{~d} x_{i}\right)$ and its avoidance function is $Z$. There is at most one $P P$ with these three properties in $\mathcal{C}(M)$.

Proof. If $\eta_{M}$ has finite factorial moment measures of all orders, then its avoidance function is $\mathbb{P}\left(\eta_{M}(B)=0\right)=\sum_{n=0}^{\infty} \frac{(-1)^{n}}{n !} \mathbb{E}\left(\xi(B)^{[n]}\right)=Z(B)$ [10, (5.4.10)]. Because the avoidance function determines the PP's law [11, 9.2.XIII], uniqueness follows.

For all $r, n_{1}, \ldots, n_{r} \in \mathbb{N}_{0}$ with $n:=\sum_{i=1}^{r} n_{i}$ and disjoint $A_{1}, \ldots, A_{r} \in \mathcal{B}^{1}$, show that

$$
\mathbb{E}\left(\prod_{i=1}^{r} \eta_{M}\left(A_{i}\right)^{\left[n_{i}\right]}\right)=\int_{\prod_{i=1}^{r} A_{i}^{n_{i}}} h_{n}\left(x_{1}, \ldots, x_{n}\right) \prod_{l=1}^{n} M\left(\mathrm{~d} x_{l}\right) .
$$

The 1-hard-core of $\eta_{M}$ and (8) imply that $\eta_{M}\left(A_{i}\right)^{\left[n_{i}\right]}=1$, if both $\eta_{M}\left(A_{i}\right)=1$ and $n_{i}=1$, and 0 else. Suppose that there is an $j \in[r]$ with $n_{j} \geq 2$. On the one hand, the $j$-th factor of the lhs of $(12$ equals 0 , whence the lhs of 12 equals zero. On the other hand, this gives an upper bound on the rhs of 12 of

$$
\left(\prod_{j \neq i \in[r]} M\left(A_{i}\right)^{n_{i}}\right) \int_{A_{j}^{n_{j}}} \underbrace{h_{n_{j}}\left(x_{1}, \ldots, x_{n_{j}}\right)}_{=0} \prod_{l=1}^{n_{j}} M\left(\mathrm{~d} x_{l}\right)=0 .
$$


The remaining case has $r=n$ and all $n_{i}=1$. Proceed by induction over $n$. If $n=1$ and $A \in \mathcal{B}^{1}$, then $\mathbb{E}\left(\eta_{M}(A)\right)=\mathbb{P}\left(\eta_{M}(A)=1\right)=M(A)=\int_{A} h_{1}(x) M(\mathrm{~d} x)$. The induction step from $(n-1)$ to $n$ is

$$
\begin{aligned}
& \mathbb{E}\left(\prod_{i=1}^{n} \eta_{M}\left(A_{i}\right)\right) \stackrel{8}{=} \mathbb{P}\left(\forall i \in[n]: \eta_{M}\left(A_{i}\right)=1\right) \\
& =\int_{A_{n}} \mathbb{P}_{x_{n}}\left(\forall i \in[n-1]: \eta_{M}\left(A_{i}\right)=1\right) M\left(\mathrm{~d} x_{n}\right) \\
& \stackrel{5 \mathrm{~b}}{=} \int_{A_{n}} \mathbb{P}\left(\forall i \in[n-1]: \eta_{M}\left(A_{i} \backslash U\left(x_{n}\right)\right)=1\right) M\left(\mathrm{~d} x_{n}\right) \\
& =\int_{A_{n}}\left(\int_{\prod_{i=1}^{n-1}\left(A_{i} \backslash U\left(x_{n}\right)\right)} h_{n-1}\left(x_{1}, \ldots, x_{n-1}\right) \prod_{i=1}^{n-1} M\left(\mathrm{~d} x_{i}\right)\right) M\left(\mathrm{~d} x_{n}\right) \\
& =\int_{A_{n}}\left(\int_{\prod_{i=1}^{n-1} A_{i}} h_{n}\left(x_{1}, \ldots, x_{n}\right) \prod_{i=1}^{n-1} M\left(\mathrm{~d} x_{i}\right)\right) M\left(\mathrm{~d} x_{n}\right) \\
& =\int_{\prod_{i=1}^{n} A_{i}} h_{n}\left(x_{1}, \ldots, x_{n}\right) \prod_{i=1}^{n} M\left(\mathrm{~d} x_{i}\right) \text {. }
\end{aligned}
$$

Let $k:=\kappa(B)$ and $\left\{A_{i}\right\}_{i=1}^{k}$ be a, possibly countable, partition of $B$ into elements of $\mathcal{B}^{1}$. If $k=\infty$, then $[k]=\mathbb{N}$. Let $\mathcal{N}_{k, n}:=\left\{\left(n_{1}, \ldots, n_{k}\right) \in \mathbb{N}_{0}^{k}\right.$ $\left.\sum_{i=1}^{k} n_{i}=n\right\}$. For $a \in \mathbb{R}^{k}$, the multinomial Chu-Vandermonde identity is

$$
\left(\sum_{i \in[k]} a_{i}\right)^{[n]}=\sum_{\left(n_{1}, \ldots, n_{k}\right) \in \mathcal{N}_{k, n}}\left(\begin{array}{c}
n \\
n_{1}, \ldots, n_{k}
\end{array}\right) \prod_{i=1}^{k} a_{i}^{\left[n_{i}\right]} .
$$

The multinomial Chu-Vandermonde identity together with 12 yields

$$
\begin{aligned}
\mathbb{E}\left(\eta_{M}(B)^{[n]}\right) & =\mathbb{E}\left(\sum_{\left(n_{1}, \ldots, n_{k}\right) \in \mathcal{N}_{k, n}}\left(\begin{array}{c}
n \\
n_{1}, \ldots, n_{k}
\end{array}\right) \prod_{i=1}^{k} \eta_{M}\left(A_{i}\right)^{\left[n_{i}\right]}\right) \\
& =\sum_{\left(n_{1}, \ldots, n_{k}\right) \in \mathcal{N}_{k, n}}\left(\begin{array}{c}
n \\
n_{1}, \ldots, n_{k}
\end{array}\right) \mathbb{E}\left(\prod_{i=1}^{k} \eta_{M}\left(A_{i}\right)^{\left[n_{i}\right]}\right) \\
& =\sum_{\left(n_{1}, \ldots, n_{k}\right) \in \mathcal{N}_{k, n}}\left(\begin{array}{c}
n \\
n_{1}, \ldots, n_{k}
\end{array}\right) \int_{\prod_{i=1}^{k} A_{i}^{n_{i}}} h_{n}\left(x_{1}, \ldots, x_{n}\right) \prod_{l=1}^{n} M\left(\mathrm{~d} x_{l}\right) \\
& =\int_{B^{n}} h_{n}\left(x_{1}, \ldots, x_{n}\right) \prod_{l=1}^{n} M\left(\mathrm{~d} x_{l}\right) .
\end{aligned}
$$

Proposition 29. If $M \in \mathcal{M}^{\text {sh }}$, then a strong 1-dependent and 1-hard-core PP with intensity $M$ exists.

Proof. Four sufficient conditions [11, 9.2.XV] jointly guarantee the existence of a simple $\mathrm{PP}$ with avoidance function $Z$. The conditions are

1. Proposition 25 implies the complete monotonicity of $Z$. 
2. Unit at $\emptyset$, i.e., $Z(\emptyset)=1$, holds trivially.

3. Continuity in space at $\emptyset$ follows from Proposition 22 combined with the fact that $\emptyset$ is a continuity set of $M$, as $M(\bar{\emptyset} \backslash \emptyset)=M(\emptyset)=0$.

4. Almost-sure bounded finiteness of the PP. Let $\left\{A_{i, n}\right\}_{i \in\left[k_{n}\right], n \in \mathbb{N}}$ be a dissecting system $\left[10\right.$, Prop A2.1.V] of $B \in \mathcal{B}^{\kappa}$. By intersecting every partition of the dissecting system with a fixed $\mathcal{B}^{1}$-partition of $B$, one may assume that the dissecting systems contains only finite $\mathcal{B}^{1}$-partitions. Let

$$
F(n, r):=\sum_{I \subseteq\left[k_{n}\right],|I| \leq r} \Delta\left(\left\{A_{i, n}\right\}_{i \in I}\right) Z\left(B \backslash \bigsqcup_{i \in I} A_{i, n}\right)
$$

By Proposition 27, $F(n, r)=1$ for $n \geq r \geq \kappa(B)$. Hence, $\lim _{r \rightarrow \infty} \lim _{n \rightarrow \infty} F(n, r)=$ 1.

Recall that the algebra $\mathcal{B}^{\kappa}$ generates the $\sigma$-algebra $\mathcal{B}$. Thus, there exists a simple $\mathrm{PP} \eta_{M}$ on $\mathcal{X}$ with avoidance function $Z$ on $\mathcal{B}^{\kappa}$. It rests to show, that the $\mathrm{PP} \eta_{M}$ is simple, strong 1-dependent, 1-hard-core and has intensity measure $M$. The characterisation in Proposition 28 shows that there is a unique extension of its law to all of $\mathcal{B}^{b}$.

Simpleness follows from the 1-hard-core. By Proposition 16, the function $Z$ is 1-multiplicative. Proposition 12 asserts strong 1-dependence.

Let $A \in \mathcal{B}^{1}$. The $\mathrm{PP} \varphi_{\bar{A}}$ on the closure $\bar{A}$ of $A$ chooses no point with probability $1-M(\bar{A})$ and one point with probability $M(\bar{A})$ distributed with the density $M(\mathrm{~d} x) / M(\bar{A})$. The avoidance functions of $\varphi_{\bar{A}}$ and $\bar{A} \eta_{M}$ coincide, because, for each $A \supseteq B \in \mathcal{B}^{b}$,

$$
\begin{aligned}
\mathbb{P}\left(\varphi_{\bar{A}}(B)=0\right) & =1-M(\bar{A})+M(\bar{A}) \int_{\bar{A} \backslash B} \frac{M(\mathrm{~d} x)}{M(\bar{A})} \\
& =1-M(\bar{A})+M(\bar{A} \backslash B)=1-M(B)=Z(B) .
\end{aligned}
$$

For a countable dense subset $S$ of $\mathcal{X}$, consider the following countable subset of $\mathcal{B}^{1}$.

$\mathcal{H}:=\{\{x \in \mathcal{X} \mid \delta(x, s) \leq \alpha$ or $\delta(x, t) \leq \alpha\} \mid s, t \in S$ with $1-3 \alpha:=\delta(s, t)<1\}$

If $\delta(x, y)<1$, then there is a closed $A \in \mathcal{H}$ containing both $x$ and $y$. Therefore,

$$
\begin{aligned}
\mathbb{P}\left(\eta_{M} \text { is not 1-hard-core }\right) & =\mathbb{P}\left(\inf \left\{\delta(x, y) \mid x, y \in \eta_{M}\right\}<1\right) \\
& =\mathbb{P}\left(\exists A \in \mathcal{H}: \eta_{M}(A) \geq 2\right) \leq \sum_{A \in \mathcal{H}} \mathbb{P}\left(\varphi_{A} \geq 2\right)=0 .
\end{aligned}
$$

For closed $A \in \mathcal{B}^{1}$ and all $A \supseteq B \in \mathcal{B}^{b}$,

$$
\mathbb{E}\left(\eta_{M}(B)\right)=\mathbb{E}\left(A \eta_{M}(B)\right)=\mathbb{E}\left(\varphi_{A}(B)\right)=\int_{B} \frac{1}{M(A)} M(\mathrm{~d} x) M(A)=M(B) .
$$

Linearity of expectations extends this to the intensity measure of $\eta_{M}$. 


\subsection{Proof of Theorem 4}

First, prove the statement only over $\mathcal{B}^{\kappa}$. The general statement over $\mathcal{B}^{b}$ follows by taking limits along sequences in $\mathcal{B}^{\kappa}$ to a limit in $\mathcal{B}^{b}$. Let $A, B \in \mathcal{B}^{\kappa}$. Assume $Z(B)>0$. Use induction over $k:=\kappa(A \cup B)$. Let $\xi \in \mathcal{C}(M)$ with avoidance function $Q$. If $Q(B)>0$, let $q(A, B):=Q(A \cup B) / Q(B)$. If $k=0$, then $A=$ $B=\emptyset$ and $q(\emptyset, \emptyset)=1=z(\emptyset, \emptyset)$. If $k>0$, then telescoping 10 restricts to the case $A \in \mathcal{B}^{1}$ and $A \cap B=\emptyset$. Let $\left\{A_{i}\right\}_{i=1}^{k}$ be a $\mathcal{B}^{1}$-partition of $A \sqcup B$. For $x \in A$, let $A(x)$ be the unique partition element containing $x$. Apply Proposition 14 twice to get

$$
\begin{aligned}
q(A, B) & =1-\int_{A} q(B, B \backslash U(x))^{-1} M(\mathrm{~d} x) \\
& =1-\int_{A} q(B, B \backslash A(x))^{-1} q(B \backslash A(x), B \backslash U(x))^{-1} M(\mathrm{~d} x)
\end{aligned}
$$

and, for $x \in A$,

$$
q(B, B \backslash A(x))=1-\int_{B \cap A(x)} q(B \backslash A(x), B \backslash A(x) \backslash U(y))^{-1} M(\mathrm{~d} y) .
$$

The second half of this proof shows that the expansions $(13)$ are well-defined. For $x \in A$ and $y \in A(x)$, one has $A(x) \subseteq U(y)$ and $\kappa(B \backslash A(x)) \leq k-1$. Hence, the inductive hypothesis applies to the integrand in $(13 \mathrm{~b}$ and the second factor of the integrand in $13 \mathrm{a}$. For $x \in A$, the inductive hypothesis implies that $Q(B \backslash A(x))>0$. Apply $(9)$ to $13 \mathrm{~b}$ ) to see that

$$
\begin{aligned}
q(B, B \backslash A(x)) & \geq 1-\int_{B \cap A(x)} z(B \backslash A(x), B \backslash A(x) \backslash U(y))^{-1} M(\mathrm{~d} y) \\
& =z(B, B \backslash A(x))
\end{aligned}
$$

Substitute this into 13a, multiply and see that this implies that $Q(B)>0$. Apply (9) to $13 \mathrm{a}$ and obtain

$$
\begin{aligned}
q(A, B) & \geq 1-\int_{A} z(B, B \backslash A(x))^{-1} z(B \backslash A(x), B \backslash U(x))^{-1} M(\mathrm{~d} x) \\
& =1-\int_{A} z(B, B \backslash U(x))^{-1} M(\mathrm{~d} x)=z(A, B) .
\end{aligned}
$$

\subsection{Proof of Theorem 6}

If $M$ has an atom of mass one at $x$, then the strong 0 -dependent $\mathrm{PP} \xi$ from Proposition 15 has $\mathbb{P}(\xi(\{x\})=0)=0$. Thus, it suffices to consider only measures with atoms smaller than one. For every $M \in \mathcal{M}^{\text {sh }} \backslash \mathcal{M}^{>}$, the avoidance function of Shearer's PP $\eta_{M}$ vanishes on some bounded Borel set and Proposition 6 follows trivially.

For every $M \notin \mathcal{M}^{\text {sh }}$, there exists $B \in \mathcal{B}^{b}$ with $Z(B, M)<0$ and $M(B)>$ 0 . Let $\bar{B}$ be the closure of $B$. Proposition 21 implies that $\lambda_{B}=\min \{\lambda \mid$ $Z(B, \lambda M) \leq 0\}, 0<\lambda_{\bar{B}} \leq \lambda_{B}<1$ and $\left.\lambda_{\bar{B}} \bar{M}\right|_{\bar{B}} \in \mathcal{M}^{\text {sh }}$. Proposition 19 asserts that, for each $\bar{B} \supseteq A \in \mathcal{B}^{b}$ and $N \leq \lambda_{B} M, Z(A, N) \geq 0$. From here on, assume that $B$ is closed with $Z(B, M)<0$. Let $\Lambda:=\lambda_{B}$.

Consider three independent PPs. 
- Proposition 15 guarantees the existence of a strong 1-dependent $\varphi \in \mathcal{C}(M)$.

- For a yet undetermined $N \in \mathcal{M}^{b}$, a (maybe non-simple) $\operatorname{Poisson}(N) \operatorname{PP}$ $\vartheta$ on $B$.

- As $\left.\Lambda M\right|_{B} \in \mathcal{M}^{\text {sh }}$, Proposition 29 guarantees the existence Shearer's PP $\eta_{\Lambda M}$

Recall that $\psi^{\bullet}$ is the simple support PP of a general PP $\psi$. The target $\mathrm{PP}$ is $\xi:=$ $(\mathcal{X} \backslash B) \varphi+\left(\eta_{\Lambda M}+\vartheta\right)^{\bullet}$. As all three component PPs are strong 1-dependent, so is $\xi$. Because $\mathbb{P}(\xi(B)=0)=\mathbb{P}\left(\eta_{\Lambda M}(B)=0, \vartheta(B)=0\right) \leq \mathbb{P}\left(\eta_{\Lambda M}(B)=0\right)=0$, the avoidance probability of $\xi$ vanishes on $B$.

To determine $N$, verify that it has finite mass on $B$ and that $M$ is the intensity of $\xi$. Let $\mathcal{A}$ be the atoms of $M$ in $B$. The diffuse domain is $\mathcal{D}:=B \backslash \mathcal{A}$. Set $\left.N\right|_{\mathcal{D}}:=\left.(1-\Lambda) M\right|_{\mathcal{D}}$, which is finite. Because $\mathcal{D} \eta_{\Lambda M}$ and $\mathcal{D} \vartheta$ are simple and independent, for each $B \supseteq A \in \mathcal{B}^{b}$,

$\mathbb{E}(\xi(\mathcal{D} \cap A))=\mathbb{E}\left(\left(\eta_{\Lambda M}+\vartheta\right)^{\bullet}(\mathcal{D} \cap A)\right)=\mathbb{E}\left(\eta_{\Lambda M}(\mathcal{D} \cap A)\right)+\mathbb{E}(\vartheta(\mathcal{D} \cap A))=M(\mathcal{D} \cap A)$.

For an atom $x \in \mathcal{A}$, let $n_{x}:=\mathbb{E}(\vartheta(\{x\}))$. The construction demands that

$$
\begin{aligned}
m_{x}:=\mathbb{E}(\xi(\{x\})) & =\mathbb{E}\left(\left(\eta_{\Lambda M}+\vartheta\right)^{\bullet}(\{x\})\right) \\
& =\mathbb{P}\left(\eta_{\Lambda M}(\{x\})=1\right)+\mathbb{P}\left(\eta_{\Lambda M}(\{x\})=0, \vartheta(\{x\}) \geq 1\right) \\
& =\mathbb{E}\left(\eta_{\Lambda M}(\{x\})\right)+Z(\{x\}, \Lambda M)(1-\mathbb{P}(\vartheta(\{x\})=0)) \\
& =\Lambda m_{x}+\left(1-m_{x}\right)\left(1-\exp \left(-n_{x}\right)\right) .
\end{aligned}
$$

Since $m_{x}<1$, then so is $\frac{(1-\Lambda) m_{x}}{1-\Lambda m_{x}}<1$ and

$$
n_{x}=-\log \left(1-\frac{(1-\Lambda) m_{x}}{1-\Lambda m_{x}}\right) \stackrel{6}{\leq} \frac{\frac{(1-\Lambda) m_{x}}{1-\Lambda m_{x}}}{1-\frac{(1-\Lambda) m_{x}}{1-\Lambda m_{x}}}=\frac{(1-\Lambda) m_{x}}{1-m_{x}}
$$

As $M(\mathcal{A}) \leq M(B)<\infty$ and all atoms of $M$ are less than one, let $m_{B}:=$ $\max \left\{m_{x} \mid x \in \mathcal{A}\right\}<1$. The finiteness of the atomic part of $N$ follows from

$$
N(\mathcal{A})=\sum_{x \in \mathcal{A}} n_{x} \leq \sum_{x \in \mathcal{A}} \frac{(1-\Lambda) m_{x}}{1-m_{x}} \leq \frac{(1-\Lambda)}{1-m_{B}} \sum_{x \in \mathcal{A}} m_{x}=\frac{(1-\Lambda)}{1-m_{B}} M(\mathcal{A})<\infty .
$$

\subsection{Intrinsic coupling and monotonicity}

Proposition 30. Let $\eta_{M}$ be Shearer's PP with intensity measure $M$. Let $p$ : $\mathcal{X} \rightarrow[0,1]$ be measurable. Define $N \in \mathcal{M}^{b}$ by $N(B):=\int_{B} p(x) M(\mathrm{~d} x)$. The independent p-thinning [11, Section 11.3] of $\eta_{M}$ has the same law as $\eta_{N}$.

Proof. Independent thinning preserves strong 1-dependence and the 1-hardcore. It also implies the intensity measure $N$. Conclude by the uniqueness from Proposition 28.

Proof of Theorem 7 . Choose the thinning in Proposition 30 with $p=\frac{\mathrm{d} N}{\mathrm{~d} M}$. This proves that $\mathcal{M}^{\text {sh }}$ and $\mathcal{M}^{>}=\mathcal{M}^{+}$are down-sets. The proofs of Proposition 15 and Theorem 6 imply that $\mathcal{M}^{\emptyset}$ and $\mathcal{M}^{0} \sqcup \mathcal{M}^{\emptyset}$ are up-sets respectively. 


\subsection{Shearer's PP is different}

Except in trivial cases (zero intensity measure, space with isolated small components), Shearer's PP differs from other well known hard-core PPs. Because a hard-core radius lower bounds a dependence radius, the difficulty is combining 1-dependence and 1-hard-core. Shearer's PP is not a Poisson PP, one of Matérn's constructions 26, 35, 36, a hard-sphere model as in Section 2.4 and is neither a determinantal nor permanental PP 6, 15. In special cases explicit constructions are possible, though. On the graph $\mathbb{Z}$ for homogeneous intensity and all radii $R$ [27, on $\mathbb{R}$ for homogeneous intensity and all radii $R$ [19] and on chordal graphs for $R=2$ and all admissible intensities [20].

The space $(\mathcal{X}, \delta)$ is $r$-connected, if each pair of points is part of a finite point sequence with consecutive pairwise distance less than $r$, and $(\mathcal{X}, \delta)$ is $r$ disconnected, if $\inf \{\delta(x, y) \mid\{x, y\} \subseteq \mathcal{X}\} \geq r$. If $(\mathcal{X}, \delta)$ is 1-disconnected, then Shearer's PP is a product BRF. For the remainder of this section, assume that $(\mathcal{X}, \delta)$ is 1-connected with diameter greater than one and that, for all $x \in \mathcal{X}$ and each neighbourhood $B$ of $x, M(B)>0$.

Proposition 31. Shearer's PP is not a Matérn-style hard-core PP.

Proof. Let $N \in \mathcal{M}^{b}$. For the extreme cases of diffuse and atomic $N$, let $\varphi$ be a Poisson $(N) \mathrm{PP}$ and a product BRF of intensity $N$ respectively. Additionally, attach iid Uniform $([0,1])$ marks to points of $\varphi$. There are disjoint $A_{1}, A_{2}, A_{3} \in$ $\mathcal{B}^{1}$ with positive $M$-measure with the diameters of $A_{1} \sqcup A_{2}$ and $A_{2} \sqcup A_{3}$ smaller than $R$ and $\delta\left(A_{1}, A_{3}\right) \geq R$. This reduces the setting to BRFs on the graph $G:=([3],\{\{1,2\},\{2,3\}\})$ with the geodesic metric $d$, inducing the metric space $([3], 2 d)$. The aim is to show that an 1-hard-core clashes with independence of the marginals at 1 and 3 . Let $\left(n_{1}, n_{2}, n_{3}\right)$ and $\left(m_{1}, m_{2}, m_{3}\right)$ be the underlying and positive target atomic intensities respectively.

For the Matérn I hard-core PP [26, 35], delete all points of $\varphi$ having at least another point at distance less than 1 . The target intensities are $m_{1}=n_{1}\left(1-n_{2}\right)$, $m_{2}=\left(1-n_{1}\right) n_{2}\left(1-n_{3}\right)$ and $m_{3}=\left(1-n_{2}\right) n_{3}$. 1-dependence demands that $n_{1}\left(1-n_{2}\right)^{2} n_{3}=m_{1} m_{3}=n_{1}\left(1-n_{2}\right) n_{3}$. This implies that $n_{2}=0$ and contradicts $m_{2}>0$.

To obtain the Matérn II hard-core PP [26, 35], delete every point $x$ of $\varphi$ whose mark $l$ fulfils $l=\max \{h \mid(y, h) \in \varphi, \delta(x, y)<1\}$. By symmetry, the comparison between the labels on neighbouring sites yields a probability of $1 / 2$ in a site's favour. The target intensities are $m_{1}=n_{1}\left(1-n_{2} / 2\right), m_{2}=n_{2}\left(1-n_{1} / 4\right)\left(1-n_{3} / 4\right)$ and $m_{3}=n_{3}\left(1-n_{2} / 2\right)$. 1-dependence demands that $n_{1}\left(1-n_{2} / 2\right)^{2} n_{3}=m_{1} m_{3}=$ $n_{1}\left(1-3 n_{2} / 4\right) n_{3}$. This implies that $n_{2}=0$, a contradiction to $m_{2}>0$.

A marked point $(x, l)$ inhibits a marked point $(y, k)$, if $\delta(x, y)<1$ and $l \leq k$. A marked point $(x, l)$ is uninhibited, if it fulfils $l=\min \{h \mid(y, h) \in \varphi, \delta(x, y)<1\}$. Iteratively, delete all inhibited points. Uninhibited points only contribute once to the deletion and every 1-connected cluster of points contains at least one uninhibited point. Hence, the deletion procedure stabilises locally almost-surely and the resulting PP is the Matérn III hard-core PP 36 with radius 1 . The target intensities are $m_{1}=n_{1}\left(1-n_{2} / 2+n_{2} n_{3} / 4\right)$ and $m_{3}=n_{3}\left(1-n_{2} / 2+n_{2} n_{1} / 4\right)$. This implies that $\left.n_{1}, n_{3} \in\right] 0,1$ [. 1-dependence demands that $n_{1} n_{3}\left(1-n_{2} / 2+\right.$ $\left.n_{2} n_{3} / 4\right)\left(1-n_{2} / 2+n_{2} n_{1} / 4\right)=m_{1} m_{3}=n_{1}\left(1-n_{2} / 4\right) n_{3}$. This is impossible, as the lhs is always bigger than the rhs.

Proposition 32. Shearer's $P P$ is not the hard-sphere model. 
Proof. Let $A, B \in \mathcal{B}^{1}$ with $A \subsetneq B$ and $0<M(A)<M(B)$. Let $h_{B}$ be the hardsphere model with fugacity $N$ on $B$. It has avoidance function $N /(1+N(B))$. Demanding equal laws for Shearer's PP of intensity $N$ and the hard-sphere model with fugacity $N$ on $A, B$ and $B \backslash A$ leads to only trivial solutions of

$$
\frac{N(B)}{1+N(B)}=M(B)=M(A)+M(B \backslash A)=\frac{N(A)}{1+N(A)}+\frac{N(B)-N(A)}{1+M(B)-M(A)} .
$$

Proposition 33. Shearer's PP is neither determinantal nor permanental.

Proof. The higher moment densities of a determinantal PP are the determinants of a matrix with entries from a bivariate, symmetric and measurable kernel $K: \mathcal{X}^{2} \rightarrow \mathbb{R}$. Consider the correlation function of $n$ points, i.e. the RadonNikodyn derivative of the $n$-th factorial moment measure of $\eta_{M}$ with respect to the $n$-fold product of $M$. It depends only on the 1-connected graph structure of the $n$ points and takes values 1 and 0 , for 1-disconnected graphs and graphs containing at least one 1-edge, respectively.

For $x \in \mathcal{X}$, this yields $\operatorname{det} K(x, x)=1$. For $\{x, y\} \subseteq \mathcal{X}$, this yields $K(x, y)=$ \pm 1 and $K(x, y)=0$ for $\delta(x, y)<1$ and $\delta(x, y) \geq 1$ respectively. For $n=3$, the graph $(\{x, y, z\},\{\{x, y\},\{y, z\}\})$ yields the contradiction $0=1-K(x, y)^{2}-$ $K(y, z)^{2}=-1$. PP.

The attraction of permanental PPs contradicts the 1-hard-core of Shearer's

\section{Proofs of the LLLs}

Lemma 34. If (1) holds, then $\mathcal{B}^{b}=\mathcal{B}^{\kappa}$.

Proof. Induction on $\operatorname{diam}(B):=\sup \{\delta(x, y) \mid x, y \in B\}$ shows that, for each $B \in \mathcal{B}^{b}, \kappa(B) \leq K^{\lfloor\operatorname{diam}(B)\rfloor}<\infty$.

Proof of Theorem 8. By (1) and Lemma $34, \mathcal{B}^{b}=\mathcal{B}^{\kappa}$. Let $A, B \in \mathcal{B}^{\kappa}$. This proof uses induction over $k:=\kappa(A \cup B)$. If $k=0$, then $A=B=\emptyset$ and $z(\emptyset, \emptyset)=1$. If $k>0$, then telescope 10 to restrict to $A \backslash B \in \mathcal{B}^{1}$. Let $\left\{A_{i}\right\}_{i=1}^{k}$ be a $\mathcal{B}^{1}$ partition of $A \cup B$. For $x \in A \cup B$, let $A(x)$ be the partition element containing $x$. Apply (9) twice to get

$$
\begin{aligned}
z(A, B) & =1-\int_{A \backslash B} z(B, B \backslash U(x))^{-1} M(\mathrm{~d} x) \\
& =1-\int_{A \backslash B} z(B, B \backslash A(x))^{-1} z(B \backslash A(x), B \backslash U(x))^{-1} M(\mathrm{~d} x)
\end{aligned}
$$

and, for $x \in A \backslash B$,

$$
z(B, B \backslash A(x))=1-\int_{B \cap A(x)} z(B \backslash A(x), B \backslash U(y))^{-1} M(\mathrm{~d} y) .
$$

For $x \in A \backslash B$ and $y \in A(x), A(x) \subseteq U(y)$, whence $\kappa(B \backslash U(y)) \leq \kappa(B \backslash$ $A(x)) \leq k-1$. Thus, the inductive hypothesis applies to the integrand of $14 \mathrm{~b}$ 
and the second factor in $14 \mathrm{a}$. Bounding the integrand of $14 \mathrm{~b}$ by the inductive hypothesis $(2 \mathrm{~b})$ leads to

$$
\begin{aligned}
z(B, B \backslash A(x)) & \geq 1-\int_{B \cap A(x)}\left(\frac{K+2}{K+1}\right)^{\kappa(U(y))} M(\mathrm{~d} y) \\
& \stackrel{1}{\geq} 1-\left(\frac{K+2}{K+1}\right)^{K} M(B \cap A(x)) \\
& \geq 1-\left(\frac{K+2}{K+1}\right)^{K} \frac{(K+1)^{K+1}}{(K+2)^{K+2}} \\
& \geq 1-\frac{1}{K+2}=\frac{K+1}{K+2} .
\end{aligned}
$$

Substituting this into the rhs of $14 \mathrm{a}$ and bounding the right factor of the integrand by the inductive hypothesis (2b) leads to

$$
\begin{aligned}
z(A, B) & \geq 1-\int_{A \backslash B} \frac{K+2}{K+1}\left(\frac{K+2}{K+1}\right)^{\kappa(U(x))} M(\mathrm{~d} x) \\
& \stackrel{1}{\geq} 1-\left(\frac{K+2}{K+1}\right)^{K+1} M(A \backslash B) \\
& \geq 1-\left(\frac{K+2}{K+1}\right)^{K+1} \frac{(K+1)^{K+1}}{(K+2)^{K+2}} \\
& =1-\frac{1}{K+2}=\frac{K+1}{K+2} .
\end{aligned}
$$

Proof of Theorem 9. By (1) and Lemma 34, $\mathcal{B}^{b}=\mathcal{B}^{\kappa}$. Theorem 9 follows from telescoping the statement $\forall A \in \mathcal{B}^{1}, B \in \mathcal{B}^{b}: z(A, B) \geq \frac{\alpha}{1+\alpha}$. Let $N:=(1+\alpha) M$. The equality $(9)$ and the monotonicity of $z$ in $M$ from Proposition 19 imply that

$$
\begin{aligned}
0 \leq z(A, B, N) & =1-\int_{A \backslash B} z(B \backslash A, B \backslash U(x), N)^{-1} N(\mathrm{~d} x) \\
& \leq 1-\int_{A \backslash B} z(B \backslash A, B \backslash U(x), M)^{-1} \frac{d N}{d M}(x) M(\mathrm{~d} x) \\
& \leq 1-(1+\alpha) \int_{A \backslash B} z(B \backslash A, B \backslash U(x), M)^{-1} M(\mathrm{~d} x) \\
& =1-(1+\alpha)(1-z(A, B, M)) .
\end{aligned}
$$

Proof of Theorem 10. Assuming that $(3 \mathrm{~b})$ holds for $A, B \in \mathcal{B}^{\kappa}$, the general case follows from a limiting argument. Let $A, B \in \mathcal{B}^{b}$ with $A \cap B=\emptyset$. Take sequences $\left(A_{n}\right)_{n \in \mathbb{N}}$ and $\left(B_{n}\right)_{n \in \mathbb{N}}$ in $\mathcal{B}^{\kappa}$ exhausting $A$ and $B$ respectively. The monotonicity of $z$ in space from Proposition 19 implies that

$$
z(A, B)=\lim _{n \rightarrow \infty} z\left(A_{n}, B_{n}\right) \geq \lim _{n \rightarrow \infty} \exp \left(-N\left(A_{n} \backslash B_{n}\right)\right)=\exp (-N(A \backslash B)) .
$$

It remains to prove $\left[3 \mathrm{~b}\right.$ ) for $A, B \in \mathcal{B}^{\kappa}$. The first part of the proof is verbatim the same as the one of Theorem 8 , leading to $(14)$. For $x \in A$, bounding the integrand in (14b) by the inductive hypothesis (3b) leads to

$$
z(B, B \backslash A(x)) \geq 1-\int_{B \cap A(x)} \exp (N((B \backslash A(x)) \cap U(y))) M(\mathrm{~d} y)
$$




$$
\begin{aligned}
& \geq 1-\int_{B \cap A(x)} \exp (N(U(y) \backslash(B \cap A(x)))) M(\mathrm{~d} y) \\
& \geq \exp (-N(B \cap A(x))) .
\end{aligned}
$$

Substituting this into the rhs of $14 \mathrm{a}$ and using the inductive hypothesis on the right factor of the integrand leads to

$$
\begin{aligned}
z(A, B) & \geq 1-\int_{A \backslash B} \exp (N(B \cap A(x))) \exp (N((B \cup U(x)) \backslash A(x))) M(\mathrm{~d} x) \\
& =1-\int_{A \backslash B} \exp (N(B \cap U(x))) M(\mathrm{~d} x) \\
& \geq 1-\int_{A \backslash B} \exp (N(U(x) \backslash(A \backslash B))) M(\mathrm{~d} x) \\
& \geq \exp (-N(A \backslash B)) .
\end{aligned}
$$

Proof of Corollary 11. Here $X=\mathbb{R}^{d}, M=\lambda \mathcal{L}$ and $N=\alpha \mathcal{L}$. For each $A \in$ $\mathcal{B}^{1}$ with $L:=\mathcal{L}(A)$, condition (4) rewrites into $\lambda L e^{\alpha(V-L)} \leq 1-e^{-\alpha L}$. The identity $\lambda e^{\alpha V}=\alpha$ simplifies this to $\alpha L e^{-\alpha L}+e^{-\alpha L} \leq 1$. The last inequality is just $1+z \leq e^{z}$ with $z:=\alpha L \geq 0$. Thus, condition (3a) holds. Conclude via Theorem 10 .

\section{Additional material}

\subsection{Association and ordering properties}

This section comments on various ordering properties of Shearer's PP with respect to factorial moment ordering and void function ordering. It also contrasts the association of the avoidance function of Shearer's PP and the hard-sphere model. Terminology is taken from [5] or [9].

Using Proposition 28 together with the bound (7), implies that all factorial and ordinary moment measures of $\eta_{M}$ are less than the ones of a Poisson PP of intensity $M$. In other words, $\eta_{M}$ is a $\alpha$-weakly sub Poisson PP.

For Shearer's PP $\eta_{M}$, Proposition 19 implies that, for disjoint $A, B \in \mathcal{B}^{b}$,

$$
\mathbb{P}\left(\eta_{M}(A \sqcup B)=0\right)=Z(A \sqcup B, M) \leq \mathbb{P}\left(\eta_{M}(A)=0\right) \mathbb{P}\left(\eta_{M}(B)=0\right) .
$$

By [9, Proposition 3.1], $\eta_{M}$ is a $\nu$-weakly sub Poisson PP, i.e., $\mathbb{P}\left(\eta_{M}(B)=0\right) \leq$ $\exp (-M(B))$ holds, for all $B \in \mathcal{B}^{b}$. Note: 9, Proposition 3.1] only demands a diffuse measure for the super-weakly Poisson direction.

In the context of Corollary 11, the avoidance function of $\eta_{\lambda \mathcal{L}}$ is bigger than the avoidance function of a homogeneous Poisson PP of intensity $\alpha$. Because $\lambda<\lambda e^{-\lambda V}=\alpha$ (as $V \geq 1$ and assuming that $\lambda \neq 0$ ), $\eta_{\lambda \mathcal{L}}$ is a $\nu$-weakly super Poisson $(\alpha)$ PP. So its avoidance function is bigger than the one of a Poisson intensity of the bigger intensity $\alpha$.

For $k \in \mathbb{N}_{0}$, let $f: \mathbb{R} \rightarrow\{0,1\}$ be the indicator function of zero and $g:=1-f$. The functions $f$ and $g$ are monotone decreasing and increasing, respectively. For each PP $\xi$ and $B \in \mathcal{B}^{b}, \mathbb{P}(\xi(B)=0)=\mathbb{E}(f(\xi(A)))$. With $a:=\mathbb{E}\left(g\left(\eta_{M}(A)\right)\right)$ and $b:=\mathbb{E}\left(g\left(\eta_{M}(B)\right)\right)$, the inequality 15 a rewrites as

$$
\mathbb{P}\left(\eta_{M}(A \sqcup B)=0\right)=\mathbb{E}\left(f\left(\eta_{M}(A \sqcup B)\right)\right)
$$




$$
\begin{aligned}
& =\mathbb{E}\left(f\left(\eta_{M}(A)\right) f\left(\eta_{M}(B)\right)\right) \\
& =\mathbb{E}\left(\left(1-g\left(\eta_{M}(A)\right)\right)\left(1-g\left(\eta_{M}(B)\right)\right)\right) \\
& =1-a-b+\mathbb{E}\left(g\left(\eta_{M}(A)\right) g\left(\eta_{M}(B)\right)\right) \\
& \leq 1-a-b+a b \\
& =\mathbb{E}\left(1-g\left(\eta_{M}(A)\right)\right) \mathbb{E}\left(1-g\left(\eta_{M}(B)\right)\right) \\
& =\mathbb{E}\left(f\left(\eta_{M}(A)\right)\right) \mathbb{E}\left(f\left(\eta_{M}(B)\right)\right) \\
& =\mathbb{P}\left(\eta_{M}(A)=0\right) \mathbb{P}\left(\eta_{M}(B)=0\right) .
\end{aligned}
$$

This implies that

$$
\frac{\mathbb{E}\left(g\left(\eta_{M}(A)\right) g\left(\eta_{M}(B)\right)\right)}{\mathbb{E}\left(g\left(\eta_{M}(A)\right)\right) \mathbb{E}\left(g\left(\eta_{M}(B)\right)\right)} \leq 0 .
$$

The extension to more than two disjoint sets via Proposition 19 is straightforward. Thus, the avoidance function of $\eta_{M}$ exhibits negative association.

For $A, B \in \mathcal{B}^{b}$, the spatial submultiplicativity of $Z(A \sqcup B,-M)$ implies that

$$
\mathbb{P}\left(h_{A \sqcup B, M}(A \sqcup B)=0\right)=\frac{1}{Z(A \sqcup B,-M)} \geq \mathbb{P}\left(h_{A, M}(A)=0\right) \mathbb{P}\left(h_{B, M}(B)=0\right) .
$$

Hence, the avoidance function of the hard-sphere model exhibits positive association.

The question whether $\eta_{M}$ is negatively associated (cf. [9, Sec 2.3]) is still open.

\subsection{Miscellaneous proofs}

The classic Chu-Vandermonde identity is

$$
(a+b)^{[n]}=\sum_{n_{a}=0}^{n}\left(\begin{array}{c}
n \\
n_{a}
\end{array}\right) a^{\left[n_{a}\right]} b^{\left[n-n_{a}\right]}=\sum_{n_{a}, n_{b}}\left(\begin{array}{c}
n \\
n_{a}, n_{b}
\end{array}\right) a^{\left[n_{a}\right]} b^{\left[n_{b}\right]} .
$$

The multinomial form in the proof of Proposition 28 follows by induction. For $k \geq 3$, one has

$$
\begin{aligned}
\left(\sum_{i \in[k]} a_{i}\right)^{[n]} & =\sum_{n_{k}=0}^{n}\left(\begin{array}{c}
n \\
n_{k}
\end{array}\right) a_{k}^{\left[n_{k}\right]}\left(\sum_{i \in[k-1]} a_{i}\right)^{\left[n-n_{k}\right]} \\
& =\sum_{n_{k}=0}^{n}\left(\begin{array}{c}
n \\
n_{k}
\end{array}\right) a_{k}^{\left[n_{k}\right]} \sum_{n_{1}, \ldots, n_{k-1}}\left(\begin{array}{c}
n-n_{k} \\
n_{1}, \ldots, n_{k-1}
\end{array}\right) \prod_{i \in[k-1]} a_{i}^{\left[n_{i}\right]} \\
& =\sum_{n_{1}, \ldots, n_{k}}\left(\begin{array}{c}
n \\
n_{1}, \ldots, n_{k}
\end{array}\right) \prod_{i=1}^{k} a_{i}^{\left[n_{i}\right]}
\end{aligned}
$$

The trivial solutions in the proof of Proposition 32 are as follows. Let $b:=$ $N(B)$ and $a:=N(A)$. The condition rewrites to

$\frac{b}{1+b}=\frac{a}{1+a}+\frac{b-a}{1+b-a}=\frac{a+a b-a^{2}+b-a+a b-a^{2}}{(1+a)(1+b-a)}=\frac{2 a b-2 a^{2}+b}{(1+a)(1+b-a)}$. 
This yields

$$
\begin{aligned}
0 & =\left(2 a b-2 a^{2}+b\right)(1+b)-b(1+a)(1+b-a) \\
& =-2 a^{2}+b(1+a)+b\left(2 a b-2 a^{2}+b\right)-b\left(1+b+a b-a^{2}\right) \\
& =2 a^{2}+b\left(a+a b-a^{2}\right) \\
& =a\left(2 a+b+b^{2}+a b\right)
\end{aligned}
$$

Thus, either $a=0$, or $a>0$ and $b^{2}+b(1+a)+2 a=0$. The latter equation has no solution with $b \geq 0$.

The determinants used in the proof of Proposition 33 are as follows. For $n=1$ and each $x \in \mathcal{X}$, this implies that $\operatorname{det} K(x, x)=1$. For $n=2$ and all $x, y \in \mathcal{X}$ with $\delta(x, y)<1$, this yields $0=1-K(x, y)^{2}$ and $K(x, y)= \pm 1$. For $n=2$ and all $x, y \in \mathcal{X}$ with $\delta(x, y) \geq 1$, this yields $1=1-K(x, y)^{2}$ and $K(x, y)=0$. For $n=3$, the graph $(\{x, y, z\},\{\{x, y\},\{y, z\}\})$ yields the contradiction

$$
0=\left|\begin{array}{ccc}
1 & K(x, y) & 0 \\
K(x, y) & 1 & K(z, y) \\
0 & K(z, y) & 1
\end{array}\right|=1-K(x, y)^{2}-K(y, z)^{2}=-1 .
$$

\subsection{Direct proof of homogeneous LLL in Euclidean space}

Direct proof of Corollary 11. Because $\mathcal{X}=\mathbb{R}^{d}$ satisfies (1), Lemma 34 asserts that $\mathcal{B}^{b}=\mathcal{B}^{\kappa}$. Thus, the proof continues from the proof of Theorem 8 at (14). Recall that $M=\lambda \mathcal{L}$. For $x \in A \backslash B$, let $l_{x}:=\mathcal{L}(B \cap A(x))$. Bounding the integrand of $(14 \mathrm{~b}$ by the inductive hypothesis leads to

$$
\begin{array}{rlr}
z(B, B \backslash A(x)) & \left.\geq 1-\int_{B \cap A(x)} \exp (\alpha \mathcal{L}(B \backslash A(x)) \cap U(y))\right) \lambda \mathcal{L}(d y) \\
& \geq 1-\int_{B \cap A(x)} \exp (\alpha \mathcal{L}(U(y) \backslash(B \cap A(x)))) \lambda \mathcal{L}(d y) \\
& =1-\lambda l_{x} \exp \left(\alpha\left(V-l_{x}\right)\right) & \\
& =1-\alpha l_{x} \exp \left(-\alpha l_{x}\right) & \\
& \geq \exp \left(-\alpha l_{x}\right) & \text { as } e^{z} \geq 1+z \\
& =\exp (-\alpha \mathcal{L}(B \cap A(x))) . &
\end{array}
$$

Let $l:=\mathcal{L}(A \backslash B)$. Substituting this into the rhs of $\sqrt{14 a}$ and using the inductive hypothesis on the right factor of the integrand leads to

$$
\begin{array}{rlrl}
z(A, B) & \geq 1-\int_{A \backslash B} \exp (\alpha \mathcal{L}(B \cap A(x))) \exp (\alpha \mathcal{L}((B \backslash A(x)) \cap U(x))) \lambda \mathcal{L}(d x) \\
& \geq 1-\int_{A \backslash B} \exp (\alpha \mathcal{L}(U(x) \backslash(A \backslash B))) \lambda \mathcal{L}(d x) \\
& =1-\lambda l \exp (\alpha(V-l)) \quad \text { as } \lambda \exp (\alpha V)=\alpha \\
& =1-\alpha l \exp (-\alpha l) & \\
& \geq \exp (-\alpha l) & \text { as } e^{z} \geq 1+z \\
& =\exp (-\alpha \mathcal{L}(A \backslash B)) . &
\end{array}
$$




\subsection{Inductive proofs}

Proposition 35. If $A \in \mathcal{B}^{1}$, then $z(A, B) \leq 1-M(A)$.

Proof. The FE (9), monotonicity $(19)$ and the upper bound $1=Z(\emptyset)$ yield

$$
z(A, B)=1-\int_{A} z(B \cap U(x), B \backslash U(x))^{-1} M(d x) \leq 1-\int_{A} 1 M(x)=1-M(A) .
$$

Proposition 36. If $A_{1}, A_{2} \in \mathcal{B}^{1}$ are disjoint and $B \in \mathcal{B}^{\kappa}$ with $\left(A_{1} \cup B\right) \cap A_{2}=\emptyset$ and $\left(A_{1} \sqcup A_{2}\right) \in \mathcal{B}^{1}$, then $z\left(A_{1}, B\right) \geq M\left(A_{2}\right)$.

Proof. Apply the FE (9), factorize and bound by 1 from Proposition 35

$$
\begin{aligned}
0 \leq z\left(A_{2}, A_{1} \cup B\right) & =1-\int_{A_{2}} z\left(A_{1} \cup B,\left(A_{1} \cup B\right) \backslash U(x)\right)^{-1} M(d x) \\
& =1-\int_{A_{2}} z\left(A_{1} \cup B, B \backslash U(x)\right)^{-1} M(d x) \\
& =1-\int_{A_{2}} z\left(A_{1}, B\right)^{-1} z(B, B \backslash U(x))^{-1} M(d x) \\
& \leq 1-\int_{A_{2}} z\left(A_{1}, B\right)^{-1} M(d x) \\
& =1-z\left(A_{1}, B\right)^{-1} M\left(A_{2}\right) .
\end{aligned}
$$

Proposition 37. For all $A, B \in \mathcal{B}^{\kappa}$ with $A \subseteq B$, if

$$
Z(B)>0 \Rightarrow Z(A)>0 \text {. }
$$

Proof. Use induction over $k:=\kappa(B \backslash A)$. If $k=0$, then $A=B$ and $Z(A)=$ $Z(B)>0$. If $k=1$, then apply the FE $(9)$ to obtain

$$
Z(B \backslash A)=Z(B)+\int_{B \backslash A} Z(B \backslash U(x)) M(d x) \geq Z(B)>0,
$$

as $M \in \mathcal{M}^{\text {sh }}$ implies that the integrand is non-negative. If $k>1$, then choose $\left\{A_{i}\right\}_{i=0}^{k}$ with $A=: A_{0} \subsetneq A_{1} \subsetneq \ldots \subsetneq A_{k}:=B$ and $\kappa\left(A_{i} \backslash A_{i-1}\right)=1$, for all $i \in[k]$. Apply the statement for the previous case $k$ times and obtain

$$
Z(A)=Z\left(A_{0}\right) \geq Z\left(A_{1}\right) \geq \ldots \geq Z\left(A_{k}\right)=Z(B)>0 .
$$

Proposition 38. For every $A, B \in \mathcal{B}^{\kappa}$,

$$
A \subseteq B \Rightarrow Z(A) \geq Z(B) .
$$

Proof. Use induction over $k:=\kappa(B \backslash A)$. If $k=0$, then $A=B$ and $Z(A)=$ $Z(B)$. If $k=1$, then apply the FE $(9)$ to obtain

$$
Z(B \backslash A)=Z(B)+\int_{B \backslash A} Z(B \backslash U(x)) M(d x) \geq Z(B),
$$

as $M \in \mathcal{M}^{\text {sh }}$ implies that the integrand is non-negative. If $k>1$, then choose $\left\{A_{i}\right\}_{i=0}^{k}$ with $A=: A_{0} \subsetneq A_{1} \subsetneq \ldots \subsetneq A_{k}:=B$ and $\kappa\left(A_{i} \backslash A_{i-1}\right)=1$, for all $i \in[k]$. Apply the statement for the previous case $k$ times and obtain

$$
Z(A)=Z\left(A_{0}\right) \geq Z\left(A_{1}\right) \geq \ldots \geq Z\left(A_{k}\right)=Z(B) .
$$


Proposition 39. If $A, B \in \mathcal{B}^{\kappa}$ with $Z(B)>0$, then

$$
z(A, B) \leq 1
$$

Proof. Without loss of generality, restrict to the case of $A \in \mathcal{B}^{1}$, telescoping otherwise. Assume that $A \cap B=\emptyset$. Apply the FE (9) to get

$$
z(A, B)=1-\int_{A} z(B, B \backslash U(x))^{-1} M(d x) \leq 1
$$

where the inequality holds if the integrand is positive. By assumption, $Z(B)>$ 0 . Thus, for every $x \in A$, also $Z(B \backslash U(x))>0$ by (17) and the integrand $z(B, B \backslash U(x))^{-1}$ is positive.

Proposition 40. For each $A, A^{\prime}, B, B^{\prime} \in \mathcal{B}^{\kappa}$, if

$$
A \subseteq A^{\prime}, B \subseteq B^{\prime}, A \cap B=A \cap B^{\prime} \Rightarrow z(A, B) \geq z\left(A^{\prime}, B^{\prime}\right) .
$$

Proof. The general case combines the following two inequalities, varying in only one of the two arguments:

$$
z(A, B) \geq z\left(A^{\prime}, B\right) \geq z\left(A^{\prime}, B^{\prime}\right)
$$

Case $B=B^{\prime}$ : Factorise and apply the upper bound with value 1 from 19 to the first factor to get

$$
z\left(A^{\prime}, B\right)=z\left(A^{\prime}, A \cup B\right) z(A, B) \leq z(A, B) .
$$

Case $A=A^{\prime}$ : Use induction over $k:=\kappa\left(A \cup B^{\prime}\right)$. If $k=0$, then $A=B=$ $B^{\prime}=\emptyset$ and $z(\emptyset, \emptyset)=1$. If $k>0$, telescope 10$)$ to restrict ourselves to the case $A \in \mathcal{B}^{1}$ and $A \cap B=A \cap B^{\prime}=\emptyset$. Let $\left(A_{i}^{\prime}\right)_{i=1}^{k}$ be a $\mathcal{B}^{1}$-partition of $A \sqcup B^{\prime}$. Derive a $\mathcal{B}^{1}$-partition $\left(A_{i}\right)_{i=1}^{k}$ of $A \sqcup B$ via $A_{i}:=A_{i}^{\prime} \cap(A \cup B)$. For $x \in A$, let $A^{\prime}(x)$ and $A(x)$ be the unique partition elements containing $x$.

For $x \in A, y \in A(x) \subseteq A^{\prime}(x), A(x) \subseteq A^{\prime}(x) \subseteq U(y)$ and $\kappa(B \backslash A(x)) \leq$ $\kappa\left(B^{\prime} \backslash A^{\prime}(x)\right) \leq k-1$. Apply the first case and the inductive hypothesis to obtain

$$
z(B \backslash A(x), B \backslash U(y)) \geq z\left(B^{\prime} \backslash A^{\prime}(x), B \backslash U(y)\right) \geq z\left(B^{\prime} \backslash A^{\prime}(x), B^{\prime} \backslash U(y)\right) .
$$

Apply the preceding inequality, twice the FE (9) and enlarge the integration domain to get

$$
\begin{aligned}
z(B \cap A(x), B \backslash A(x)) & =1-\int_{B \cap A(x)} z(B \backslash A(x), B \backslash U(y))^{-1} M(d y) \\
& \geq 1-\int_{B \cap A(x)} z\left(B^{\prime} \backslash A^{\prime}(x), B^{\prime} \backslash U(y)\right)^{-1} M(d y) \\
& \geq 1-\int_{B^{\prime} \cap A^{\prime}(x)} z\left(B^{\prime} \backslash A^{\prime}(x), B^{\prime} \backslash U(y)\right)^{-1} M(d y) \\
& =z\left(B^{\prime} \cap A^{\prime}(x), B^{\prime} \backslash A^{\prime}(x)\right) .
\end{aligned}
$$


Again, for each $x \in A \backslash B^{\prime}$, the preceding inequality for the first factor and the inductive hypothesis for the second factor imply that

$$
\begin{aligned}
z(B, B \backslash U(x)) & =z(B \cap A(x), B \backslash A(x)) z(B \backslash A(x), B \backslash U(x)) \\
& \geq z\left(B^{\prime} \cap A^{\prime}(x), B^{\prime} \backslash A^{\prime}(x)\right) z\left(B^{\prime} \backslash A^{\prime}(x), B^{\prime} \backslash U(x)\right) \\
& =z\left(B^{\prime}, B^{\prime} \backslash U(x)\right) .
\end{aligned}
$$

Another two applications of the FE (9) and the preceding inequality yield

$$
\begin{aligned}
z(A, B)=1-\int_{A} z(B, & \backslash U(x))^{-1} M(d x) \\
& \geq 1-\int_{A} z\left(B^{\prime}, B^{\prime} \backslash U(x)\right)^{-1} M(d x)=z\left(A, B^{\prime}\right) .
\end{aligned}
$$

\section{Acknowledgements}

The author acknowledges the support of the VIDI project "Phase transitions, Euclidean fields and random fractals", NWO 639.032.916, while working at the VU Amsterdam. The author thanks Marie-Colette van Lieshout for discussions about general PP theory and the anonymous reviewers and editor for their helpful comments.

\section{References}

[1] Jon Aaronson, David Gilat, and Michael Keane. On the structure of 1dependent Markov chains. J. Theoret. Probab., 5(3):545-561, 1992.

[2] Jon Aaronson, David Gilat, Michael Keane, and Vincent de Valk. An algebraic construction of a class of one-dependent processes. Ann. Probab., 17(1):128-143, 1989.

[3] Noga Alon and Joel H. Spencer. The probabilistic method. WileyInterscience Series in Discrete Mathematics and Optimization. John Wiley \& Sons Inc., Hoboken, NJ, third edition, 2008. With an appendix on the life and work of Paul Erdős.

[4] A. J. Baddeley, M. N. M. van Lieshout, and J. Møller. Markov properties of cluster processes. Adv. in Appl. Probab., 28(2):346-355, 1996.

[5] Bartłomiej Błaszczyszyn and Dhandapani Yogeshwaran. Stochastic Geometry, Spatial Statistics and Random Fields: Models and Algorithms, chapter Clustering Comparison of Point Processes, with Applications to Random Geometric Models, pages 31-71. Springer International Publishing, Cham, 2015.

[6] Alexei Borodin. Determinantal point processes. In The Oxford handbook of random matrix theory, pages 231-249. Oxford Univ. Press, Oxford, 2011.

[7] Erik I. Broman. One-dependent trigonometric determinantal processes are two-block-factors. Ann. Probab., 33(2):601-609, 2005. 
[8] Robert M. Burton, Marc Goulet, and Ronald Meester. On 1-dependent processes and $k$-block factors. Ann. Probab., 21(4):2157-2168, 1993.

[9] Bartłomiej Błaszczyszyn and D. Yogeshwaran. On comparison of clustering properties of point processes. Adv. in Appl. Probab., 46(1):1-20, 032014.

[10] D. J. Daley and D. Vere-Jones. An introduction to the theory of point processes. Vol. I. Probability and its Applications (New York). SpringerVerlag, New York, second edition, 2003. Elementary theory and methods.

[11] D. J. Daley and D. Vere-Jones. An introduction to the theory of point processes. Vol. II. Probability and its Applications (New York). Springer, New York, second edition, 2008. General theory and structure.

[12] V. de Valk. The maximal and minimal 2-correlation of a class of 1dependent 0-1 valued processes. Israel J. Math., 62(2):181-205, 1988.

[13] V. de Valk. Hilbert space representations of $m$-dependent processes. Ann. Probab., 21(3):1550-1570, 1993.

[14] R. L. Dobrushin. Estimates of semi-invariants for the Ising model at low temperatures. In Topics in statistical and theoretical physics, volume 177 of Amer. Math. Soc. Transl. Ser. 2, pages 59-81. Amer. Math. Soc., Providence, RI, 1996.

[15] Nathalie Eisenbaum. Stochastic order for alpha-permanental point processes. Stochastic Process. Appl., 122(3):952-967, 2012.

[16] P. Erdős and L. Lovász. Problems and results on 3-chromatic hypergraphs and some related questions. In Infinite and finite sets (Colloq., Keszthely, 1973; dedicated to P. Erdös on his 60th birthday), Vol. II, pages 609-627. Colloquia Mathematica Societatis János Bolyai, Vol. 10. North-Holland, Amsterdam, 1975.

[17] Roberto Fernández and Aldo Procacci. Cluster expansion for abstract polymer models. New bounds from an old approach. Comm. Math. Phys., 274(1):123-140, 2007.

[18] Roberto Fernández, Aldo Procacci, and Benedetto Scoppola. The analyticity region of the hard sphere gas. Improved bounds. J. Stat. Phys., 128(5):1139-1143, 2007.

[19] Christoph Hofer-Temmel. Shearer's point process and the hard-sphere model in one dimension. Preprint, 2015+.

[20] Christoph Hofer-Temmel and Florian Lehner. Clique trees of infinite, locally finite chordal graphs. Submitted, 2015.

[21] Alexander E. Holroyd. One-dependent coloring by finitary factors. arXiv:1411.1463, 2014+.

[22] Svante Janson. Runs in $m$-dependent sequences. Ann. Probab., 12(3):805818, 1984. 
[23] Tommy R. Jensen and Bjarne Toft. Graph coloring problems. WileyInterscience Series in Discrete Mathematics and Optimization. John Wiley \& Sons Inc., New York, 1995. A Wiley-Interscience Publication.

[24] R. Kotecký and D. Preiss. Cluster expansion for abstract polymer models. Comm. Math. Phys., 103(3):491-498, 1986.

[25] T. M. Liggett, R. H. Schonmann, and A. M. Stacey. Domination by product measures. Ann. Probab., 25(1):71-95, 1997.

[26] Bertil Matérn. Spatial variation: Stochastic models and their application to some problems in forest surveys and other sampling investigations. Meddelanden Fran Statens Skogsforskningsinstitut, Band 49, Nr. 5, Stockholm, 1960 .

[27] Pierre Mathieu and Christoph Temmel. $k$-independent percolation on trees. Stochastic Process. Appl., 122(3):1129-1153, 2012.

[28] S. Miracle-Solé. On the theory of cluster expansions. Markov Process. Related Fields, 16(2):287-294, 2010.

[29] O. Penrose. Convergence of fugacity expansions for classical systems. In T. A. Bak, editor, Statistical Mechanics: Foundations and Applications, pages 101-109, New York-Amsterdam, 1967.

[30] T. Rolski and R. Szekli. Stochastic ordering and thinning of point processes. Stochastic Process. Appl., 37(2):299-312, 1991.

[31] David Ruelle. Statistical mechanics: Rigorous results. W. A. Benjamin, Inc., New York-Amsterdam, 1969.

[32] Alexander D. Scott and Alan D. Sokal. The repulsive lattice gas, the independent-set polynomial, and the Lovász local lemma. J. Stat. Phys., 118(5-6):1151-1261, 2005.

[33] J. B. Shearer. On a problem of Spencer. Combinatorica, 5(3):241-245, 1985.

[34] A. Soshnikov. Determinantal random point fields. Uspekhi Mat. Nauk, $55(5(335)): 107-160,2000$.

[35] Dietrich Stoyan and Helga Stoyan. On one of Matérn's hard-core point process models. Math. Nachr., 122:205-214, 1985.

[36] J. Teichmann, F. Ballani, and K.G. van den Boogaart. Generalizations of Matérn's hard-core point processes. Spatial Statistics, 3(0):33 - 53, 2013.

[37] Christoph Temmel. Shearer's measure and stochastic domination of product measures. J. Theoret. Probab., 27(1):22-40, 2014.

[38] M. N. M. van Lieshout and A. J. Baddeley. A nonparametric measure of spatial interaction in point patterns. Statist. Neerlandica, 50(3):344-361, 1996. 